\title{
Structure of microphytoplankton community and environmental variables in a macrotidal estuarine complex, São Marcos Bay, Maranhão - Brazil
}

\author{
Lisana Furtado Cavalcanti ${ }^{1}$, Andrea Christina Gomes Azevedo-Cutrim ${ }^{2}$, Amanda Lorena Lima \\ Oliveira ${ }^{1}$, Jordana Adorno Furtado ${ }^{1}$, Bethânia de Oliveira Araújo ${ }^{1}$, Ana Karoline Duarte-dos- \\ Santos Sál, Francinara Santos Ferreira ${ }^{I}$, Nágela Gardênia Rodrigues Santos ${ }^{l}$, Francisco José \\ Silva Dias ${ }^{1}$, Marco Valério Jansen Cutrim ${ }^{1}$ (i)
}

\author{
${ }^{1}$ Departamento de Oceanografia e Limnologia da Universidade Federal do Maranhão \\ (Av. dos Portugueses, 1966 - Bacanga - 65080-805 - São Luís - MA Brazil) \\ ${ }^{2}$ Departamento de Química e Biologia da Universidade Estadual do Maranhão \\ (Cidade Universitária Paulo VI, Av. Lourenço Vieira da Silva, nº 1000 - Jardim São Cristovão - 65055-310 - São Luís - MA - Brazil) \\ *Corresponding author: cutrim@ufma.br
}

\section{Abstract}

São Marcos Bay is an estuarine complex with semidiurnal tides that can reach more than $7 \mathrm{~m}$ during equinoctial spring tides. It is situated in the second largest Brazilian coastline and is subjected to continuous human activities holding an important port complex of Latin America. In order to contribute to the knowledge about the structure of the phytoplankton in a macrotidal systems, this study aims to evaluate the phytoplankton community and its relationship with environmental conditions in the São Marcos Bay. Five surveys in 2010 and 2011 were carried out on four sampling points during the rainy and dry seasons. Samples were taken during the flood and ebb phases at neap tides. Hydrological parameters were correlated with biological data (phytoplankton composition, abundance and biomass) using statistical analysis. The phytoplankton biomass (chlorophyll $a$ ) characterized the bay as quite productive $\left(10.43 \pm 7.62 \mathrm{mg} \mathrm{m}^{-3}\right)$ with the nanophytoplankton as the dominant fraction. Seasonal variation was observed in phytoplankton abundance with higher values $\left(34,262 \pm 18,422\right.$ cells $\left.\mathrm{L}^{-1}\right)$ in the rainy season. Diatoms were the most important phytoplankton group, pointing out Nitzschia sp., Diploneis weissflogii and Synedra sp. as the dominant species. This study revealed that the composition of phytoplankton community was mainly influenced by the local dynamics, governed by macrotides, and precipitation regime that mostly contributed to the seasonal fluctuations of the environmental conditions, such as salinity, dissolved oxygen, and nutrients.

Descriptors: Macrotidal estuaries, Phytoplankton abundance, Chlorophyll $a$, Nutrients.

\section{INTRODUCTION}

Transitional and coastal water bodies such as estuaries and coastal lagoons are complex environments that link freshwater and marine systems (Bazin et al., 2014). They also go through continuous changes in response to their dynamic and natural processes. Because of their hydrodynamic characteristics, estuarine systems are among the most productive and resourceful aquatic ecosystems around the world (Sathicq et al., 2017).

Submitted on: 23/November/2017

Approved on: 18/September/2018

http://dx.doi.org/10.1590/S1679-87592018021906603
These systems receive rich riverine supplies of nutrients and provide important ecosystem services (e.g., food production, nutrient cycling), becoming crucial to the maintenance of marine life and humanity (Costanza et al., 1997; Attrill and Rundle, 2002). Furthermore, these ecosystems are excellent ecological study sites due to their biotic and abiotic mechanisms varying over space and time, which control the distribution of organisms in the whole system (Sin et al., 2015). However, due to their proximity to land, estuaries have been more vulnerable to anthropogenic activities, such as domestic and industrial effluent contamination, which, under extreme conditions, can affect their productivity and consequently the water quality (Carstensen et al., 2011; Cloern et al., 2014). 
In macrotidal zones, physical processes control the system dynamics with large amounts of organic and inorganic compounds being reworked and transported within the estuary (Burford et al., 2008). In turbid estuaries under strong mixing conditions, tidal force is the most dominant factor, which can vary over time (diurnally, fortnightly or seasonally) (Azhikodan and Yokoyama, 2016). Wind force also promotes continuous vertical mixing of the water column allowing the translocation of oxygen-saturated water and protecting proximate coastal water bodies from eutrophication since macrotidal currents decrease the response of primary production to enhanced nutrient inputs (Cloern, 2001).

The biological indicators are commonly used to assess the water quality detecting changes in the biotic structure of the communities over spatial and temporal scales (Katsiapi et al., 2016). Phytoplankton plays a crucial role in the aquatic environment because they are the dominant primary producers and balance the overall food web dynamics (Katz et al., 2004; Chai et al., 2016). In addition to holding ecological services, biomass, composition, and community structure of phytoplankton can be considered an efficient bio-indicator of water quality as their distribution strongly correlates with several factors (e.g., physical, chemical, and biological) as well as interactions among them (Paerl et al., 2003; 2010). This variability may be reflected in population dynamics, especially those of phytoplankton populations thriving in coastal systems.

Studies addressed the dynamics of phytoplankton community in estuaries along the north coast of Maranhão - Brazil, especially in the São Marcos Bay, are scarce (Teixeira et al., 1988; Lavôr-Fernandes, 1988; Azevedo et al., 2008; Rodrigues and Cutrim, 2010; Duarte-dos-Santos et al., 2017). The analysis of the seasonal and tidal distribution of phytoplankton community and the complex relationships of these distribution patterns with environmental factors in the São Marcos Bay can identify potential indicative species of ecological conditions as well as provide relevant scientific evidence to water quality assessment and pollution control. Thus, the aim of this present study is to contribute to the knowledge about the structure of the phytoplankton community in terms of composition, abundance, chlorophyll $a$ and evaluate its relationship with environmental conditions in a macrotidal estuarine complex of the São Marcos Bay.

\section{MATERIAL AND METHODS}

\section{STUDY AREA}

São Marcos Bay is a wide estuarine complex located in the Maranhão state (Figure 1), holding the second largest Brazilian coastline with about $640 \mathrm{~km}$ of extension. It has a semidiurnal macrotide with tidal range that can reach more than $8 \mathrm{~m}$ in some zones during equinoctial spring tides and has tidal currents with velocities higher than $1.1 \mathrm{~m} \mathrm{~s}^{-1}$ (maximum tidal currents of $2.42 \mathrm{~m} \mathrm{~s}^{-1}$ ) (GonzálezGorbeña et al., 2015).

São Marcos Bay together with São José Bay forms the geologic unit known as the Maranhense Gulf which encloses estuaries, straits, inlets, small rivers, and many islands. The bay is surrounded by an extensive mangrove area, such as a large mangrove island, named Caranguejos Island, that is situated in its southern half portion (SouzaFilho et al., 2005; El-Robrini et al., 2006). The three main rivers of the watershed are Pindaré, Mearim, and Grajaú, whose waters discharge at the southernmost region of the São Marcos Bay being responsible for the suspended solid loads, primarily rich in clay and silt (annual average of $\sim 250 \mathrm{mg} \mathrm{L}^{1-}$ ) (Morais, 1977).

The entrance of the bay presents a width of $\sim 55 \mathrm{~km}$, which narrows to $1.5 \mathrm{~km}$ at the intersection of Pindare and Mearim rivers. The bay has a central channel with depths up to $90 \mathrm{~m}$, which works as a waterway for the second most important port complex of Latin America including São Luís port, Itaqui port, Ponta da Madeira terminal, and Alumar terminal (Amaral and Alfredini, 2010). Ponta da Madeira terminal is undergoing an expansion to increase its export capacity to 235 millions of tons per year of iron mineral, becoming the port with the largest volume of cargo in Brazil (González-Gorbeña et al., 2015).

With regard to the climate in the Maranhão state, where São Marcos Bay is located, it is tropical and humid with equatorial air mass influence and intrinsic characteristics such as: high temperatures throughout the year $\left(27^{\circ} \mathrm{C}\right.$ $31^{\circ} \mathrm{C}$ ) and two distinct seasonal periods strongly marked by the precipitation: rainy season (January-June) and dry season (July-December) (Rios, 2001).

\section{FIELD SAMPLING}

Five surveys were carried out during the rainy season (April 2010, January 2011, March 2011) and the dry season (August 2010, November 2010) at four sampling points (P1 - P3 with average depth up to $22 \mathrm{~m}$ and P4 with 


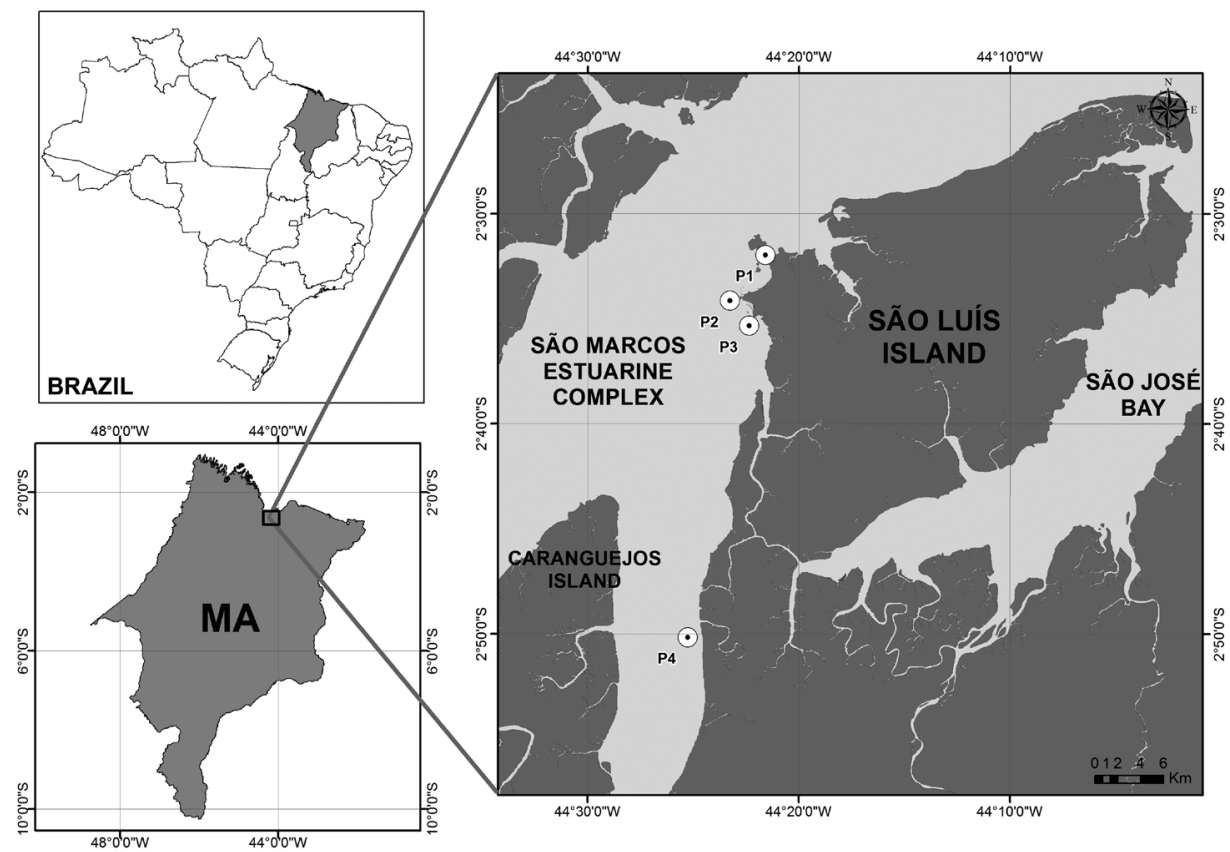

Figure 1. Position of the sampling sites and location of the estuarine complex of the São Marcos Bay, Maranhão Brazil.

5 to $10 \mathrm{~m}$; González-Gorbeña et al., 2015) along the São Marcos Bay (Figure 1). All the samples were taken during the flood and ebb tides at neap tide, comprising a total of forty samples $(n=40)$.

\section{METEOROLOGICAL AND HYDROLOGICAL ANALYSIS}

Hydrological variables were measured in situ, including temperature $\left({ }^{\circ} \mathrm{C}\right)$, salinity, $\mathrm{pH}$, dissolved oxygen $\left(\mathrm{ml} \mathrm{L}^{-1}\right)$ and oxygen saturation (\%), using a multiparametric probe (Hanna 9828). From the temperature and salinity data, we constructed a TS spread diagram, in which the plane T-S represented the water masses distribution using predetermined intervals of salinity and temperature (Miranda, 1985). The performance area for each TS pair was set according to its depth and grid point. After establishing the thermohaline indices for each water type, the mixing straight method, initially described by Mamayev (1975), was used to obtain the percentage of each water type present in a representative sample of a mixture in the stationary condition. In estuarine systems, and particularly in shallow layers, the properties of temperature and salinity are not stationary. Nevertheless, this method was used considering that the timescales involved in the variations of temperature and salinity were larger than the duration of the hydrographic surveys.
The application of this method requires the identification of thermohaline indices $\left(\mathrm{T}_{1}, \mathrm{~S}_{1}\right.$ and $\left.\mathrm{T}_{2}, \mathrm{~S}_{2}\right)$, which is usually performed using the T-S spread diagram. The percentage quantities of each water type $\left(\mathrm{m}_{1}, \mathrm{~m}_{2}\right)$ in a given sample with the temperature $\mathrm{T}$ and salinity $\mathrm{S}$ are obtained by resolution of the linear system illustrated in the following equation:

$$
\begin{aligned}
& m_{1} T_{1}+m_{2} T_{2}=m T \\
& m_{1} S_{1}+m_{2} S_{2}=m T \\
& m_{1}+m_{2}=1
\end{aligned}
$$

In addition, water transparency (Secchi depth) was measured by using a Secchi disc and turbidity (NTU) by a turbidity meter (Lamotte, 2020). For nutrient analysis, 2 liters of water were collected at the surface layer (50 $\mathrm{cm}$ depth) using a van Dorn bottle. Quantification of $\mathrm{NH}_{4}^{+}, \mathrm{NO}_{2}^{-}, \mathrm{NO}_{3}^{-}$, and $\mathrm{PO}_{4}^{-3}$ followed the methodologies described in "Standard Methods for Water and Wastewater" (APHA, 2012). The 30 years historical average precipitation in São Luís city (São Luís Island São Marcos Bay) and the wind speed for sampling months were obtained from the National Institute of Meteorology - INMET (http//www.inmet.gov.br). 


\section{MiCROPHYTOPLANKTON COMPOSITION}

To analyze qualitatively the microphytoplankton composition in the São Marcos Bay, sampling was conducted with plankton net (mesh size $45 \mu \mathrm{m}$ ). Three-minute horizontal surface hauls were carried out in order to collect the biological material. After the collection, samples were preserved with $4 \%$ formalin. In the laboratory, identification was based on the specialized phytoplankton literature. Thus, microalgae have been identified at the lowest possible taxonomic level. AlgaeBase was used to classify and update the taxa information (Guiry and Guiry, 2017).

\section{Phytoplankton abundance AND biomass}

To analyze quantitatively the phytoplankton community (phytoplankton abundance and biomass), surface water samples were collected using van Dorn bottle.

Samples were fixed with a Lugol's iodine solution to determine the phytoplankton abundance, which followed the Utermöhl method (Ferrario et al., 1995). The phytoplankton cells were counted using inverted microscopes (ZEISS Axiovert 100) with 400x magnification. At least 100 fields were counted in the deposit algae contained in $10 \mathrm{~mL}$ in each sample. In addition, the number of cells was applied to the equation of Villafañe and Reid (1995), expressing results as unit of cells per liter (cells L $\mathrm{L}^{-1}$ ).

To determine the phytoplankton biomass, estimated as size-fractionated chlorophyll $a$ concentration, surface water samples were filtered onto $47 \mathrm{~mm}$ Whatman GF/F filters $(0.7$ $\mu \mathrm{m}$ porosity). In order to separate the microphytoplankton $(>20 \mu \mathrm{m})$ from the nanophytoplankton $(<20 \mu \mathrm{m})$ fraction, each water sample was filtered through $20 \mu \mathrm{m}$ net. With a volume of $250 \mathrm{~mL}$, the chlorophyll $a$ filters were dried and stored in a freezer $\left(-18^{\circ} \mathrm{C}\right)$ until the chlorophyll pigment extraction using spectrophotometric method (UNESCO, 1966) in accordance with Parsons and Strickland (1963).

\section{DATA ANALYSIS}

Two Way Analysis of Variance (ANOVA - Two Way) was performed in order to determine the significant differences $(p<0.05)$ of the hydrological and biological variables among seasonal periods and tides. To analyze the structure of the microphytoplankton community, some indexes were applied: a) the Constancy index, on which the taxa recorded in more than $50 \%$ of the samples was considered "RESIDENT," "VISITOR" when it was recorded in $25-50 \%$ of the samples and
"ACCIDENTAL" when recorded in less than $25 \%$ of the samples (Dajoz, 1983); b) diversity index of Shannon $\left(H^{\prime}\right)$ (Shannon, 1948), evenness $(J)$ (Pielou, 1966), richness $(S)$ (Margalef, 1958) and dominance $(Y)$ (Sun et al., 2004) indexes, which were calculated based on the following equations:

Shannon index

$$
H^{\prime}=-\sum_{i=1}^{s} \mathrm{Pi} \log _{2} \times \mathrm{Pi} \quad \mathrm{Pi}=N i / N
$$

Pielou evenness index

$$
J=H^{\prime} / \log _{2} S
$$

Margalef richness index

$$
S=(s-1) / \ln \cdot N
$$

Dominance index

$$
Y=\left(n_{i} / N\right) x f i
$$

where $N i$ is the individual amount of the species organism, $N$ is the total individual amount, $S$ is the total species at any sampling point, and $f_{i}$ is the individual frequency in the samples.

The non-metric multidimensional scaling (nMDS) and cluster analysis, based on the Euclidian distance, were applied to determine the dissimilarity of sampling sites with respect to the abundance of dominant species, precipitation, and salinity (square root transformed). SIMPER analysis was used to find out the discriminating species between the seasons, where it was used the Bray-Curtis similarity coefficient to determine the similarity of dominant species through their cell abundance, defined by the dominance index. Principal Component Analysis (PCA) was computed based on a matrix formed by precipitation, wind speed, hydrological data, chlorophyll $a$ concentrations, and phytoplankton abundance in order to explore the relationship among them. The data used were standardized to normalize their distribution. In addition, Pearson's Correlation Analysis was applied to correlate the phytoplankton abundance in relation to different physical-chemical factors analyzed. Data analyses were carried out using the software IBM SPSS Statistics 24.0, STATISTIC 10.0 and PRIMER 6.0. 


\section{RESULTS}

\section{MeTEOROLOGICAL AND HYDOLOGICAL} VARIABLES

Precipitation showed an annual average of 2290.2 $\mathrm{mm}$, varying from $1755.6 \mathrm{~mm}$ in 2010 to 2713.3 in 2011. The historical average precipitation data and wind speed revealed two well-defined seasonal periods: the rainy season spanning from January to June, which can extend until July, with the highest precipitation in April $2011(621.7 \mathrm{~mm})$ and the lowest wind speed $\left(2.1 \mathrm{~m} \mathrm{~s}^{-1}\right)$ registered in January 2011. The second is the dry season spanning from July to December, where September and October did not register any precipitation value while the wind speed revealed the highest values in November 2010 $\left(4.5 \mathrm{~m} \mathrm{~s}^{-1}\right)$ (Figure 2).

Seasonal and tidal variations of hydrological variables in the São Marcos Bay during the study period are shown in Table 1. In general, water temperature ranged from $28.4{ }^{\circ} \mathrm{C}$ to $30.24{ }^{\circ} \mathrm{C}$ (overall mean $29.19 \pm 0.52{ }^{\circ} \mathrm{C}$ ) and the highest value was recorded during flood tide in the rainy season. Salinity ranged from 20.7 to 37.4 (overall mean $31.71 \pm 4.48$ ) with higher values being recorded in the dry season during ebb tide (Table 1). Significant differences $(p<0.01)$ were observed only between seasonal periods (rainy and dry seasons) in terms of water temperature and salinity.

The TS diagram pointed out two water masses in the São Marcos Bay (2010-2011) based on the criteria adopted by Dias et al. (2013) (Figure 3). The water masses were well characterized seasonally, with the presence of warmer waters $\left(\mathrm{T}>29^{\circ} \mathrm{C}\right)$ with lower salinity $(\mathrm{S}<30)$ compatible with the river water mass during the rainy season, mainly through the months of March 2010 and April 2011. During the dry season, the thermohaline indices were characteristic of coastal water with a marked increase in salinity $(31<\mathrm{S}<37)$. In January, a mixing between the two water masses was the predominant feature.

The dissolved oxygen varied from $3.08 \mathrm{mg} \mathrm{L}^{-1}$ to $6.5 \mathrm{mg}$ $\mathrm{L}^{-1}$ (overall mean $4.38 \pm 1.06 \mathrm{mg} \mathrm{L}^{-1}$ ) revealing a seasonal pattern with significant differences $(p<0.01)$ between rainy and dry seasons with higher values observed in the dry season and ebb tide. A similar trend was observed in relation to the oxygen saturation rate, which ranged from $40 \%$ to $86 \%$ (overall mean $57.9 \pm 13.94 \%$ ) (Table 1 ). No significant difference $(p>0.05)$ was observed between tidal periods (ebb tide and flood tide) in terms of dissolved oxygen and oxygen saturation.

Water transparency varied from $6 \mathrm{~cm}$ to $77 \mathrm{~cm}$ (overall mean $39 \pm 16 \mathrm{~cm}$ ) with higher values recorded during ebb tide in the rainy season (Table 1). A significant difference occurred between flood and ebb tides $(p<0.05)$. Turbidity ranged from 14 NTU to 390 NTU (overall mean 89.90 \pm 73.33 NTU) with higher values recorded in the rainy season and significant difference $(p<0.01)$ between tidal periods. In general, water transparency and turbidity did not show significant differences $(p>0.05)$ between dry and rainy seasons. The $\mathrm{pH}$ values presented higher than 7.0 in $95 \%$ of the samples ranging from 6.7 to 8.15 (overall mean 7.65 \pm 0.32 ) with no significant differences $(p>0.05)$ between seasons and tidal periods.

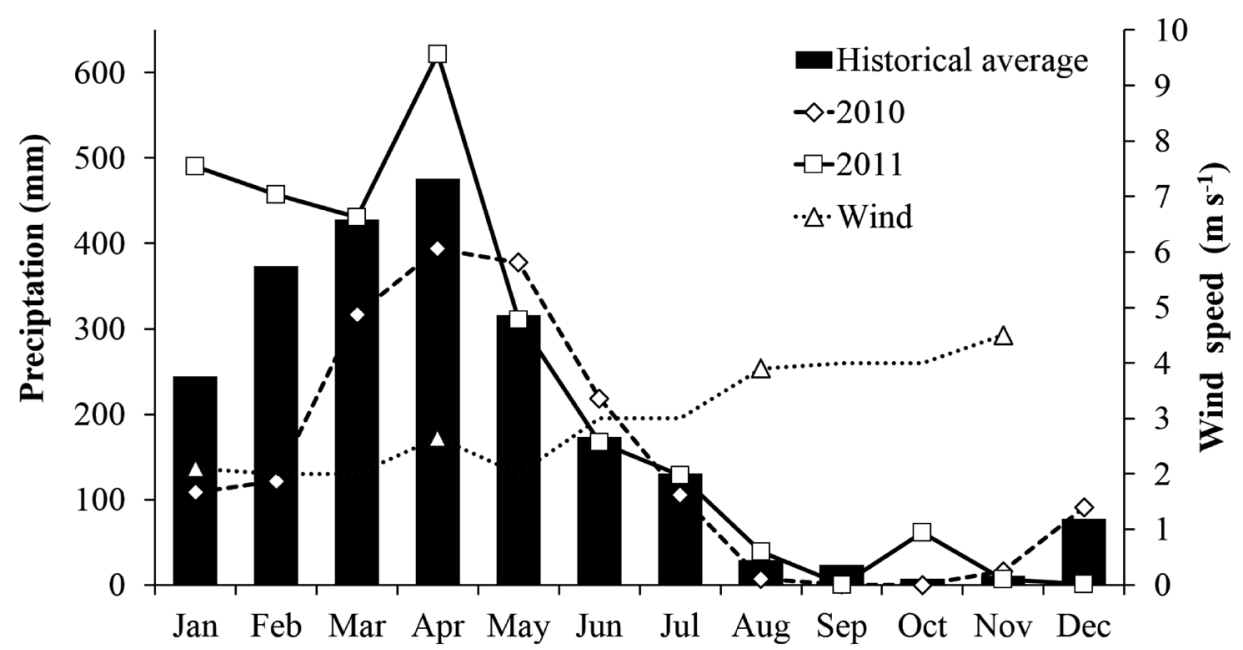

Figure 2. Historical average of precipitation (based on the last 30 years), total monthly precipitation registered in 2010 and 2011 and wind speed for the sampling months. 
Table 1. Seasonal and tidal variation of hydrological variables with mean values, standard deviation (SD) and minimum and maximum (Min/Max) in the São Marcos Bay.

\begin{tabular}{|c|c|c|c|c|c|c|c|c|}
\hline \multirow{3}{*}{$\begin{array}{l}\text { Hydrological } \\
\text { Variables }\end{array}$} & \multicolumn{4}{|c|}{ Rainy } & \multicolumn{4}{|c|}{ Dry } \\
\hline & \multicolumn{2}{|c|}{ Flood } & \multicolumn{2}{|c|}{ Ebb } & \multicolumn{2}{|c|}{ Flood } & \multicolumn{2}{|c|}{ Ebb } \\
\hline & Mean \pm SD & Min/Max & Mean \pm SD & Min/Max & Mean \pm SD & Min/Max & Mean \pm SD & $\operatorname{Min} / \operatorname{Max}$ \\
\hline Temperature $\left({ }^{\circ} \mathrm{C}\right)$ & $29.45 \pm 0.61$ & $28.56 / 30.24$ & $29.42 \pm 0.46$ & $28.72 / 30.0$ & $28.74 \pm 0.19$ & $28.4 / 29.04$ & $28.92 \pm 0.25$ & $28.6 / 29.4$ \\
\hline Salinity & $28.91 \pm 4.05$ & $20.7 / 34.78$ & $29.45 \pm 3.95$ & $21.09 / 34.8$ & $35.40 \pm 1.81$ & $31.76 / 37.3$ & $35.63 \pm 1.85$ & $32.07 / 37.4$ \\
\hline $\begin{array}{l}\text { Dissolved } \\
\text { Oxygen }\left(\mathrm{ml} \mathrm{L}^{-1}\right)\end{array}$ & $3.55 \pm 0.37$ & $3.08 / 4.5$ & $3.69 \pm 0.28$ & $3.4 / 4.16$ & $5.42 \pm 0.68$ & $4.5 / 6.3$ & $5.65 \pm 0.68$ & $4.7 / 6.5$ \\
\hline $\begin{array}{l}\text { Oxygen } \\
\text { Saturation (\%) }\end{array}$ & $47.33 \pm 5.03$ & $40 / 60$ & $48.58 \pm 4.10$ & $40 / 55$ & $71.25 \pm 9.07$ & $59 / 83$ & $74.38 \pm 9.02$ & $62 / 86$ \\
\hline $\begin{array}{l}\text { Secchi } \\
\text { depth }(\mathrm{cm})\end{array}$ & $35 \pm 13$ & $6 / 50$ & $43 \pm 23$ & $8 / 77$ & $42 \pm 9$ & $27 / 56$ & $36 \pm 15$ & $22 / 72$ \\
\hline Turbidity (NTU) & $91.29 \pm 101.35$ & $32 / 390$ & $74.08 \pm 51.85$ & $14.8 / 190$ & $102.22 \pm 67.11$ & $62.1 / 260$ & $72.50 \pm 37.57$ & $29.9 / 130$ \\
\hline $\mathrm{pH}$ & $7.66 \pm 0.44$ & $6.7 / 8.15$ & $7.7 \pm 0.35$ & $7.0 / 8.11$ & $7.52 \pm 0.18$ & $7.3 / 7.8$ & $7.69 \pm 0.13$ & $7.43 / 7.8$ \\
\hline $\mathrm{NH}_{4}^{+}\left(\mu \mathrm{mol} \mathrm{L}^{-1}\right)$ & $0.33 \pm 0.17$ & $0.07 / 0.61$ & $0.31 \pm 0.12$ & $0.17 / 0.61$ & $0.50 \pm 0.30$ & $0.15 / 0.98$ & $0.16 \pm 0.06$ & $0.09 / 0.25$ \\
\hline $\mathrm{NO}_{2}-\left(\mu \mathrm{mol} \mathrm{L} \mathrm{L}^{-1}\right)$ & $0.10 \pm 0.03$ & $0.06 / 0.16$ & $0.11 \pm 0.05$ & $0.05 / 0.26$ & $0.33 \pm 0.74$ & $0.03 / 2.16$ & $0.11 \pm 0.06$ & $0.06 / 0.26$ \\
\hline $\mathrm{NO}_{3}-\left(\mu \mathrm{mol} \mathrm{L} \mathrm{L}^{-1}\right)$ & $1.16 \pm 0.05$ & $1.08 / 1.24$ & $1.06 \pm 0.35$ & $0.13 / 1.35$ & $1.19 \pm 0,05$ & $1.10 / 1.27$ & $1.23 \pm 0.04$ & $1.13 / 1.29$ \\
\hline $\mathrm{PO}_{4}^{-3}\left(\mu \mathrm{mol} \mathrm{L}^{-1}\right)$ & $1.32 \pm 0.11$ & $0.03 / 0.46$ & $0.23 \pm 0.04$ & $0.17 / 0.33$ & $0.23 \pm 0.10$ & $0.03 / 0.34$ & $0.25 \pm 0.04$ & $0.17 / 0.33$ \\
\hline
\end{tabular}

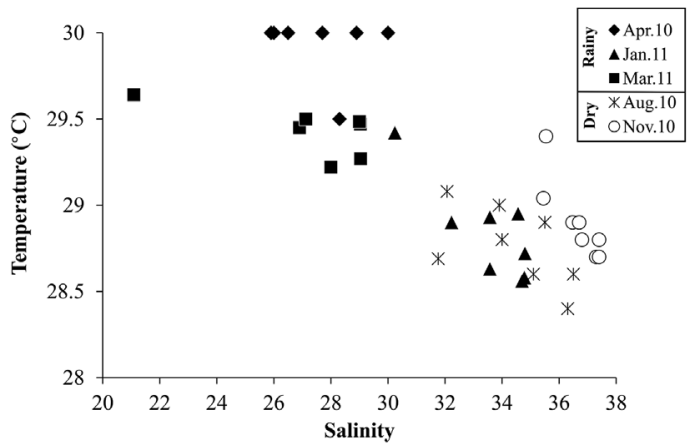

Figure 3. $T$ x $S$ Diagram for the samplings in the 4 sites at São Marcos Bay-MA in 2010 and 2011.

The concentrations of $\mathrm{NH}_{4}^{+}$ion varied from $0.07 \mu \mathrm{mol}$ $\mathrm{L}^{-1}$ to $0.98 \mu \mathrm{mol} \mathrm{L}^{-1}$ (overall mean $0.32 \pm 0.20 \mu \mathrm{mol} \mathrm{L}^{-1}$ ) with higher values being recorded in the dry season during the flood tide. In a similar way, the concentrations of $\mathrm{NO}_{2}^{-}$ showed the highest values recorded in dry season during the flood tide and ranged from $0.034 \mu \mathrm{mol} \mathrm{L}^{-1}$ to 2.16 $\mu \mathrm{mol} \mathrm{L}{ }^{-1}$ (overall mean $0.15 \pm 0.33 \mu \mathrm{mol} \mathrm{L}^{-1}$ ). However, the highest value $\left(1.36 \mu \mathrm{mol} \mathrm{L}^{-1}\right)$ of $\mathrm{NO}_{3}^{-}$was recorded in the rainy season during the ebb tide (overall mean $1.15 \pm 0.20$ $\left.\mu \mathrm{mol} \mathrm{L}{ }^{-1}\right)$. In this study, the nitrogen compounds did not show significant differences $(p>0.05)$ between seasons and tidal periods. The concentrations of $\mathrm{PO}_{4}^{-3}$ varied from 0.02 $\mu \mathrm{mol} \mathrm{L} \mathrm{L}^{-1}$ to $0.46 \mu \mathrm{mol} \mathrm{L}^{-1}$ (overall mean $0.26 \pm 0.08 \mu \mathrm{mol}$ $\mathrm{L}^{-1}$ ) with higher values observed in the rainy season during the flood tide, however, these values were not significant $(p>0.05)$ regarding the seasonal and tidal variations.

\section{MiCROPHYTOPLANKTON COMPOSITION}

From the qualitative analysis of the microphytoplankton composition, a total of 178 species were identified including 7 varieties and 3 forms (Table 2). Six divisions were distinguished: Bacillariophyta (90.45\%), Miozoa (5.62\%), Cyanobacteria (1.12\%), Chlorophyta (1.69\%), Euglenophyta $(0.56 \%)$ and Charophyta $(0.56 \%)$.

The largest group was Bacillariophyta including 68 genera and 161 taxa. The principal genus of this division was Chaetoceros (18 taxa) followed by Coscinodiscus (11 taxa), Nitzschia (10 taxa) and Actinoptychus (8 taxa). Miozoa was the second most common group with 6 genera and 10 taxa with Tripos (4 taxa) being the dominant genus.

Seasonal variation of microphytoplankton showed a number of 156 taxa identified in the rainy season and 140 in the dry season. In both seasons, diatoms were the more abundant group. During the rainy season, there were 140 diatoms $(89.74 \%), 10$ dinoflagellates $(6.41 \%)$, 2 cyanobacteria $(1.28 \%), 3$ chlorophytes $(1.92 \%)$ and 1 charophyte $(0.64 \%)$. By comparison, the dry season included 130 diatoms $(92.86 \%), 7$ dinoflagellates $(5.00 \%)$, 1 cyanobacteria $(0.71 \%)$ and 1 chlorophyte $(0.71 \%)$. Euglenophytes were observed only in the rainy season with one taxon $(0.71 \%)$. 
Table 2. List of taxa identified in microphytoplankton samples collected in the São Marcos Bay with percentage of occurrence $(\%)(n=40)$. Note: $\mathrm{TO}=$ Total Occurrence $(\mathrm{n}=40)$, classification by Constancy Index $(\mathrm{CI})$ with the categories: $\mathrm{R}=$ resident, $\mathrm{V}=$ visitor, and $\mathrm{A}=$ accidental, and occurrence by season.

\begin{tabular}{|c|c|c|c|c|c|c|c|c|c|}
\hline \multirow[t]{2}{*}{ Taxonomy Category } & \multirow{2}{*}{$\begin{array}{l}\text { TO } \\
(\%)\end{array}$} & \multirow[t]{2}{*}{ CI } & \multicolumn{2}{|c|}{$\begin{array}{c}\text { Occurence by } \\
\text { season }\end{array}$} & \multirow[t]{2}{*}{ Taxonomy Category } & \multirow{2}{*}{$\begin{array}{l}\text { TO } \\
(\%)\end{array}$} & \multirow[t]{2}{*}{ CI } & \multicolumn{2}{|c|}{$\begin{array}{c}\text { Occurence by } \\
\text { season }\end{array}$} \\
\hline & & & Dry & Rainy & & & & Dry & Rainy \\
\hline Cyanobacteria & & & & & Chaetoceros aequatorialis & 15 & A & $*$ & $*$ \\
\hline Oscillatoria spp. & 53 & $\mathrm{R}$ & $*$ & $*$ & C. affinis & 38 & $\mathrm{~V}$ & $*$ & $*$ \\
\hline Spirulina sp. & 3 & A & & $*$ & C. atlanticus & 3 & A & & $*$ \\
\hline Euglenophyta & & & & & C. atlanticus f. audax & 10 & A & $*$ & $*$ \\
\hline Euglena sp. & 3 & A & & $*$ & C. brevis & 5 & A & & $*$ \\
\hline Miozoa & & & & & C. coarctatus & 8 & A & $*$ & $*$ \\
\hline Ceratium sp. & 5 & A & $*$ & $*$ & C. compressus & 15 & A & $*$ & $*$ \\
\hline Peridinium sp. & 8 & A & $*$ & * & C. curvisetus & 13 & A & * & $*$ \\
\hline Prorocentrum sp. & 5 & A & & * & C. didymus & 5 & A & & $*$ \\
\hline Protoperidinium conicum & 13 & A & $*$ & $*$ & C. gracilis & 8 & A & $*$ & \\
\hline Protoperidinium spp. & 40 & $\mathrm{~V}$ & * & * & C. lorenzianus & 40 & $\mathrm{~V}$ & $*$ & $*$ \\
\hline Pyrophacus steinii & 10 & A & $*$ & $*$ & C. pendulus & 20 & A & $*$ & $*$ \\
\hline Tripos furca & 20 & A & $*$ & $*$ & C. peruvianus & 55 & $\mathrm{R}$ & * & $*$ \\
\hline T. fusus & 5 & A & & * & C. simplex & 25 & $\mathrm{~V}$ & $*$ & $*$ \\
\hline T. lineatus & 33 & $\mathrm{~V}$ & $*$ & * & C. subtilis & 3 & A & & $*$ \\
\hline T. macroceros & 3 & A & & $*$ & C. subtilis var. abnormis & 53 & $\mathrm{R}$ & $*$ & $*$ \\
\hline Bacillariophyta & & & & & C. teres & 5 & A & & $*$ \\
\hline Achnanthes brevipes & 3 & A & $*$ & & Chaetoceros sp. & 5 & A & & $*$ \\
\hline Actinocyclus curvatulus & 3 & A & & * & Coscinodiscopsis jonesiana & 3 & A & $*$ & \\
\hline Actinocyclus sp. & 3 & A & $*$ & & Coscinodiscus asteromphalus & 40 & $\mathrm{~V}$ & $*$ & $*$ \\
\hline Actinoptychus annulatus & 85 & $\mathrm{R}$ & $*$ & $*$ & C. centralis & 70 & $\mathrm{R}$ & * & $*$ \\
\hline A. aster & 5 & A & & $*$ & C. concinnus & 23 & A & $*$ & $*$ \\
\hline A. minutus & 5 & A & & $*$ & C. curvatulus & 3 & A & & $*$ \\
\hline A. octonarius & 10 & A & $*$ & $*$ & C. gigas & 33 & $\mathrm{~V}$ & $*$ & $*$ \\
\hline A. senarius & 78 & $\mathrm{R}$ & $*$ & $*$ & C. granii & 15 & A & $*$ & $*$ \\
\hline A. splendens & 60 & $\mathrm{R}$ & $*$ & $*$ & C. oculus-iridis & 98 & $\mathrm{R}$ & $*$ & $*$ \\
\hline A. vulgaris & 10 & A & & $*$ & C. radiatus & 85 & $\mathrm{R}$ & $*$ & $*$ \\
\hline Actinoptychus sp. & 10 & A & $*$ & $*$ & C. rothii & 60 & $\mathrm{R}$ & * & $*$ \\
\hline Alveus marinus & 3 & A & $*$ & & Coscinodiscus sp. & 28 & $\mathrm{~V}$ & * & $*$ \\
\hline Amphora sp. & 3 & A & & $*$ & Craticula cuspidata & 8 & A & $*$ & $*$ \\
\hline Asterionellopsis glacialis & 58 & $\mathrm{R}$ & $*$ & * & Cyclotella meneghiniana & 10 & A & $*$ & $*$ \\
\hline Asteromphalus sp. & 3 & A & $*$ & & C. striata & 43 & $\mathrm{~V}$ & $*$ & $*$ \\
\hline Aulacodiscus & 3 & A & $*$ & & C. stylorum & 60 & $\mathrm{R}$ & $*$ & $*$ \\
\hline margaritaceus & & A & & & Cylindrotheca closterium & 23 & A & $*$ & $*$ \\
\hline Bacillaria paxillifera & 63 & $\mathrm{R}$ & $*$ & $*$ & Diploneis bombus & 20 & A & * & $*$ \\
\hline Bacteriastrum delicatulum & 35 & V & $*$ & $*$ & D. gruendleri & 30 & $\mathrm{~V}$ & $*$ & $*$ \\
\hline B. hyalinum & 45 & $\mathrm{~V}$ & * & $*$ & D. interrupta & 5 & A & $*$ & \\
\hline Bellerochea malleus & 80 & $\mathrm{R}$ & * & $*$ & D. weissflogii & 13 & A & $*$ & $*$ \\
\hline Biddulphia biddulphiana & 3 & A & $*$ & & Ditylum brightwellii & 98 & $\mathrm{R}$ & $*$ & $*$ \\
\hline B. tridens & 8 & A & * & $*$ & Entomoneis alata & 35 & $\mathrm{~V}$ & $*$ & $*$ \\
\hline Caloneis permagna & 38 & $\mathrm{~V}$ & $*$ & $*$ & Entomoneis sp. & 3 & A & $*$ & \\
\hline Cerataulina sp. & 3 & A & $*$ & & Eupodiscus antiquus & 18 & A & $*$ & $*$ \\
\hline
\end{tabular}




\begin{tabular}{|c|c|c|c|c|}
\hline \multirow[t]{2}{*}{ Taxonomy Category } & \multirow{2}{*}{$\begin{array}{l}\text { TO } \\
(\%)\end{array}$} & \multirow[t]{2}{*}{ CI } & \multicolumn{2}{|c|}{$\begin{array}{l}\text { Occurence by } \\
\text { season }\end{array}$} \\
\hline & & & Dry & Rainy \\
\hline E. radiatus & 10 & A & & $*$ \\
\hline Eupodiscus sp. & 3 & A & $*$ & \\
\hline Fragilaria sp. & 8 & A & $*$ & \\
\hline Frustulia interposita & 33 & $\mathrm{~V}$ & $*$ & * \\
\hline F. rhomboides & 15 & A & $*$ & $*$ \\
\hline Grammatophora marina & 5 & A & $*$ & \\
\hline G. oceanica & 5 & A & $*$ & $*$ \\
\hline Guinardia flaccida & 13 & A & $*$ & $*$ \\
\hline Gyrosigma attenuatum & 10 & A & & * \\
\hline G. balticum & 83 & $\mathrm{R}$ & $*$ & * \\
\hline G. hippocampus & 13 & A & $*$ & * \\
\hline Gyrosigma sp. & 3 & A & & $*$ \\
\hline Hantzschia amphioxys & 3 & A & & $*$ \\
\hline Helicotheca tamesis & 88 & $\mathrm{R}$ & $*$ & * \\
\hline Hemiaulus indicus & 3 & A & & $*$ \\
\hline Leptocylindrus danicus & 18 & A & $*$ & $*$ \\
\hline L. minimus & 3 & A & & $*$ \\
\hline Lithodesmium undulatum & 88 & $\mathrm{R}$ & $*$ & $*$ \\
\hline Lyrella lyra & 3 & A & $*$ & \\
\hline Melchersiela hexagonalis & 50 & $\mathrm{~V}$ & * & * \\
\hline Melosira moniliformis & 23 & A & $*$ & $*$ \\
\hline M. nummuloides & 73 & $\mathrm{R}$ & $*$ & $*$ \\
\hline Navicula sp. & 13 & A & $*$ & $*$ \\
\hline Neocalyptrella robusta & 10 & A & & $*$ \\
\hline Nitzschia fasciculata & 23 & A & $*$ & $*$ \\
\hline$N$. longa & 18 & A & $*$ & $*$ \\
\hline N. longissima & 20 & A & $*$ & $*$ \\
\hline N. longissima f. parva & 3 & A & $*$ & \\
\hline N. obtusa & 5 & A & & $*$ \\
\hline $\begin{array}{l}\text { N. obtusa var. } \\
\text { scalpelliformis }\end{array}$ & 85 & $\mathrm{R}$ & $*$ & $*$ \\
\hline N. palea & 8 & A & * & $*$ \\
\hline N. sigma & 48 & $\mathrm{~V}$ & * & $*$ \\
\hline N. tryblionella & 5 & A & $*$ & $*$ \\
\hline Nitzschia $\mathrm{sp}$ & 18 & A & * & $*$ \\
\hline Odontella aurita & 55 & $\mathrm{R}$ & * & * \\
\hline O. longicruris & 60 & $\mathrm{R}$ & $*$ & $*$ \\
\hline O. turgida & 15 & A & $*$ & $*$ \\
\hline Odontella spp. & 23 & A & $*$ & $*$ \\
\hline Paralia sulcata & 60 & $\mathrm{R}$ & $*$ & $*$ \\
\hline Petroneis humerosa & 13 & A & $*$ & $*$ \\
\hline Pleurosigma angulatum & 10 & A & $*$ & $*$ \\
\hline P. formosum & 3 & $\mathrm{~A}$ & $*$ & \\
\hline
\end{tabular}

\begin{tabular}{|c|c|c|c|c|}
\hline \multirow{2}{*}{ Taxonomy Category } & \multirow{2}{*}{$\begin{array}{l}\text { TO } \\
(\%)\end{array}$} & \multirow{2}{*}{ CI } & \multicolumn{2}{|c|}{$\begin{array}{c}\text { Occurence by } \\
\text { season }\end{array}$} \\
\hline & & & Dry & Rainy \\
\hline P. normanii & 8 & A & $*$ & $*$ \\
\hline Pleurosigma spp. & 50 & $\mathrm{~V}$ & $*$ & $*$ \\
\hline Pleurosira laevis & 3 & A & $*$ & \\
\hline Podocystis adriatica & 3 & A & $*$ & \\
\hline Proboscia alata & 3 & A & & $*$ \\
\hline Psammodictyon constrictum & 8 & A & $*$ & $*$ \\
\hline P. panduriforme & 8 & A & $*$ & $*$ \\
\hline Pseudo-nitzschia pungens & 30 & $\mathrm{~V}$ & $*$ & $*$ \\
\hline Pseudo-nitzschia spp. & 28 & $\mathrm{~V}$ & $*$ & \\
\hline Pseudosolenia calcar-avis & 10 & A & $*$ & $*$ \\
\hline Rhaphoneis amphiceros & 50 & $\mathrm{~V}$ & $*$ & $*$ \\
\hline Rhizosolenia hebetata & 8 & A & $*$ & $*$ \\
\hline R. imbricata & 8 & A & & $*$ \\
\hline R. imbricata var. shrubsolei & 3 & A & & $*$ \\
\hline R. setigera & 70 & $\mathrm{R}$ & * & $*$ \\
\hline R. styliformis & 5 & A & & $*$ \\
\hline Skeletonema costatum & 68 & $\mathrm{R}$ & * & $*$ \\
\hline S. tropicum & 75 & $\mathrm{R}$ & $*$ & $*$ \\
\hline Surirella fastuosa & 5 & A & $*$ & $*$ \\
\hline S. febigeri & 45 & $\mathrm{~V}$ & $*$ & $*$ \\
\hline S. gemma & 25 & $\mathrm{~V}$ & $*$ & $*$ \\
\hline S. minuta & 3 & A & $*$ & \\
\hline Synedra ulna & 30 & $\mathrm{~V}$ & $*$ & $*$ \\
\hline S. ulna var. ulna & 28 & $\mathrm{~V}$ & $*$ & $*$ \\
\hline Synedra sp. & 20 & $\mathrm{~V}$ & $*$ & $*$ \\
\hline Tabularia fasciculata & 45 & $\mathrm{~V}$ & $*$ & $*$ \\
\hline Terpsinoe americana & 8 & A & $*$ & $*$ \\
\hline T. musica & 30 & $\mathrm{~V}$ & $*$ & $*$ \\
\hline Thalassionema frauenfeldii & 100 & $\mathrm{R}$ & $*$ & $*$ \\
\hline T. nitzschioides & 35 & $\mathrm{~V}$ & $*$ & $*$ \\
\hline $\begin{array}{l}\text { T. nitzschioides var. } \\
\text { capitulatum }\end{array}$ & 5 & A & $*$ & $*$ \\
\hline $\begin{array}{l}\text { T. nitzschioides var. } \\
\text { claviforme }\end{array}$ & 3 & A & & $*$ \\
\hline $\begin{array}{l}\text { T.nitzschioides var. } \\
\text { lanceolatum }\end{array}$ & 3 & A & & $*$ \\
\hline Thalassionema $\mathrm{sp}$. & 20 & A & & $*$ \\
\hline Thalassiosira eccentrica & 80 & $\mathrm{R}$ & $*$ & $*$ \\
\hline T. leptopus & 85 & $\mathrm{R}$ & $*$ & $*$ \\
\hline T. lineata & 13 & A & $*$ & $*$ \\
\hline T. rotula & 13 & A & $*$ & $*$ \\
\hline T. simonsenii & 3 & A & & $*$ \\
\hline T. subtilis & 98 & $\mathrm{R}$ & $*$ & $*$ \\
\hline
\end{tabular}




\begin{tabular}{|c|c|c|c|c|}
\hline \multirow[t]{2}{*}{ Taxonomy Category } & \multirow{2}{*}{$\begin{array}{l}\text { TO } \\
(\%)\end{array}$} & \multirow[t]{2}{*}{ CI } & \multicolumn{2}{|c|}{$\begin{array}{c}\text { Occurence by } \\
\text { season }\end{array}$} \\
\hline & & & Dry & Rainy \\
\hline Thalassiosira $\mathrm{sp}$. & 38 & $\mathrm{~V}$ & $*$ & $*$ \\
\hline Triceratium dubium & 3 & A & $*$ & \\
\hline T. favus & 90 & $\mathrm{R}$ & $*$ & $*$ \\
\hline T. favus f. quadrata & 48 & $\mathrm{~V}$ & $*$ & $*$ \\
\hline T. pentacrinus & 3 & A & & $*$ \\
\hline T. robertsianum & 5 & A & & $*$ \\
\hline Triceratium $\mathrm{sp}$. & 15 & A & $*$ & $*$ \\
\hline Trieres mobiliensis & 100 & $\mathrm{R}$ & $*$ & $*$ \\
\hline T. regia & 100 & $\mathrm{R}$ & $*$ & $*$ \\
\hline T. sinensis & 60 & $\mathrm{R}$ & $*$ & $*$ \\
\hline Tryblionella angustata & 3 & A & $*$ & \\
\hline $\begin{array}{l}\text { Tryblioptychus } \\
\text { cocconeiformis }\end{array}$ & 23 & A & $*$ & $*$ \\
\hline Zygoceros ehrenbergii & 93 & $\mathrm{R}$ & $*$ & $*$ \\
\hline \multicolumn{5}{|l|}{ Chlorophyta } \\
\hline Oedogonium sp. & 3 & A & & $*$ \\
\hline Dimorphococcus sp. & 33 & $\mathrm{~V}$ & $*$ & $*$ \\
\hline Scenedesmus sp. & 5 & A & & $*$ \\
\hline \multicolumn{5}{|l|}{ Charophyta } \\
\hline Staurastrum sp. & 3 & A & & $*$ \\
\hline
\end{tabular}

According to the Constancy Index analysis (Table 2), $62.36 \%$ of the species were classified as ACCIDENTAL (98 diatoms; 8 dinoflagellates; 1 cyanobacteria; 2 chlorophytes; 1 charophyte; 1 euglenophyte), $19.66 \%$ as RESIDENT (34 diatoms; 1 cyanobacteria), and $17.98 \%$ as VISITORS (29 diatoms; 2 dinoflagellates; 1 chlorophyte).

A total of 17 diatoms showed high occurrence with a percentage varying between $80 \%$ and $100 \%$ of total occurrence. Trieres mobiliensis, Trieres regia, and Thalassionema frauenfeldii were the most frequent diatoms, as they were registered in $100 \%$ of the samples $(\mathrm{n}=40)($ Table 2$)$.

\section{Phytoplankton abundance and Chloro- PHYLL A CONCENTRATION}

Phytoplankton abundance was lower in the dry season $\left(25,097 \pm 9,244\right.$ cells $\left.L^{-1}\right)$ than in the rainy season $\left(34,262 \pm 18,422\right.$ cells $\left.\mathrm{L}^{-1}\right)$ with significant seasonal difference $(p<0.05)$. In relation to tidal periods, the cell abundance was significantly higher $(p<0.01)$ in flood tide $\left(35,289 \pm 18,953\right.$ cells $\left.L^{-1}\right)$ than in ebb phase $(25,903 \pm 10,731$ cells $\mathrm{L}^{-1}$ ) (Figure 4). Only diatoms were recorded through the counting of phytoplankton cells. Based on the cell abundance, the dominance index $(Y)$ revealed 8 diatoms as the dominant species, which Nitzschia sp., Synedra sp., and Diploneis weissflogii showed higher abundance in both seasons (Table 3 ). The rainy season registered higher numbers of species; nevertheless, some species existed throughout the entire study period.

Diversity was lower in the dry season $(0.81 \pm 0.15$ bits cell $\left.{ }^{-1}\right)$ and ebb tide $\left(0.79 \pm 0.15\right.$ bits cell $\left.^{-1}\right)$, and higher in the rainy season $\left(0.83 \pm 0.14\right.$ bits cell $\left.^{-1}\right)$ and flood tide $\left(0.86 \pm 0.13\right.$ bits cell $\left.^{-1}\right)$. Likewise, richness was lower in the dry season $(2.82 \pm 0.70)$ and ebb tide $(2.82 \pm 0.76)$, and higher in the rainy season $(3.00 \pm 0.79)$ as well as flood tide (3.03 \pm 0.75 ), but some species (e.g. T. mobiliensis, T. regia, and T. frauenfeldii) occurred throughout both seasons. Evenness was lower in the rainy season $(0.94 \pm 0.05)$ and flood tide $(0.94 \pm 0.05)$, and higher in the dry season $(0.96 \pm 0.03)$ and ebb tide $(0.96 \pm 0.04)$ (Figure 4). However, no significant differences $(p>0.05)$ were observed between tidal periods and seasons in terms of diversity, richness, and evenness.

Chlorophyll $a$ concentration was lower in the dry season $\left(9.85 \pm 8.02 \mathrm{mg} \mathrm{m}^{-3}\right)$ and ebb tide $(9.85 \pm 5.11 \mathrm{mg}$ $\left.\mathrm{m}^{-3}\right)$ and higher in the rainy season $\left(10.81 \pm 7.50 \mathrm{mg} \mathrm{m}^{-3}\right)$ and flood tide (11.01 $\left.\pm 9.62 \mathrm{mg} \mathrm{m}^{-3}\right)$ (Figure 5). However, no significant differences $(p>0.05)$ were observed between tidal periods and seasons. Concerning the contribution of phytoplankton size class, nanophytoplankton was dominant $(90 \%$ of total samples) with high values in the rainy season $\left(7.59 \pm 4.59 \mathrm{mg} \mathrm{m}^{-3}\right)$ during flood tide $\left(7.36 \pm 6.07 \mathrm{mg} \mathrm{m}^{-3}\right)$, followed by microphytoplankton ( $10 \%$ of total samples) which was higher in the dry season $\left(4.33 \pm 3.88 \mathrm{mg} \mathrm{m}^{-3}\right)$ during ebb tide $\left(3.68 \pm 4.08 \mathrm{mg} \mathrm{m}^{-3}\right)$.

\section{STATISTICAL ANALYSIS}

The non-metric multidimensional (nMDS) twodimensional plot (stress 0.07) based on the abundance of dominant species, precipitation and salinity was done from cluster analysis (Euclidian distance). This analysis confirmed the formation of three major groups according to seasonality, with no overlapping between them (Figure 6). Among the three major assemblages of sampling months, groups I and III arranged mainly samples from the rainy season, except November (flood tide). In contrast, group II comprised exclusively samples from dry season (August - ebb tide, August flood tide, and November - ebb tide).

To visualize the contribution of dominant species, in terms of cell abundance (cells $\mathrm{L}^{-1}$ ), associated with 

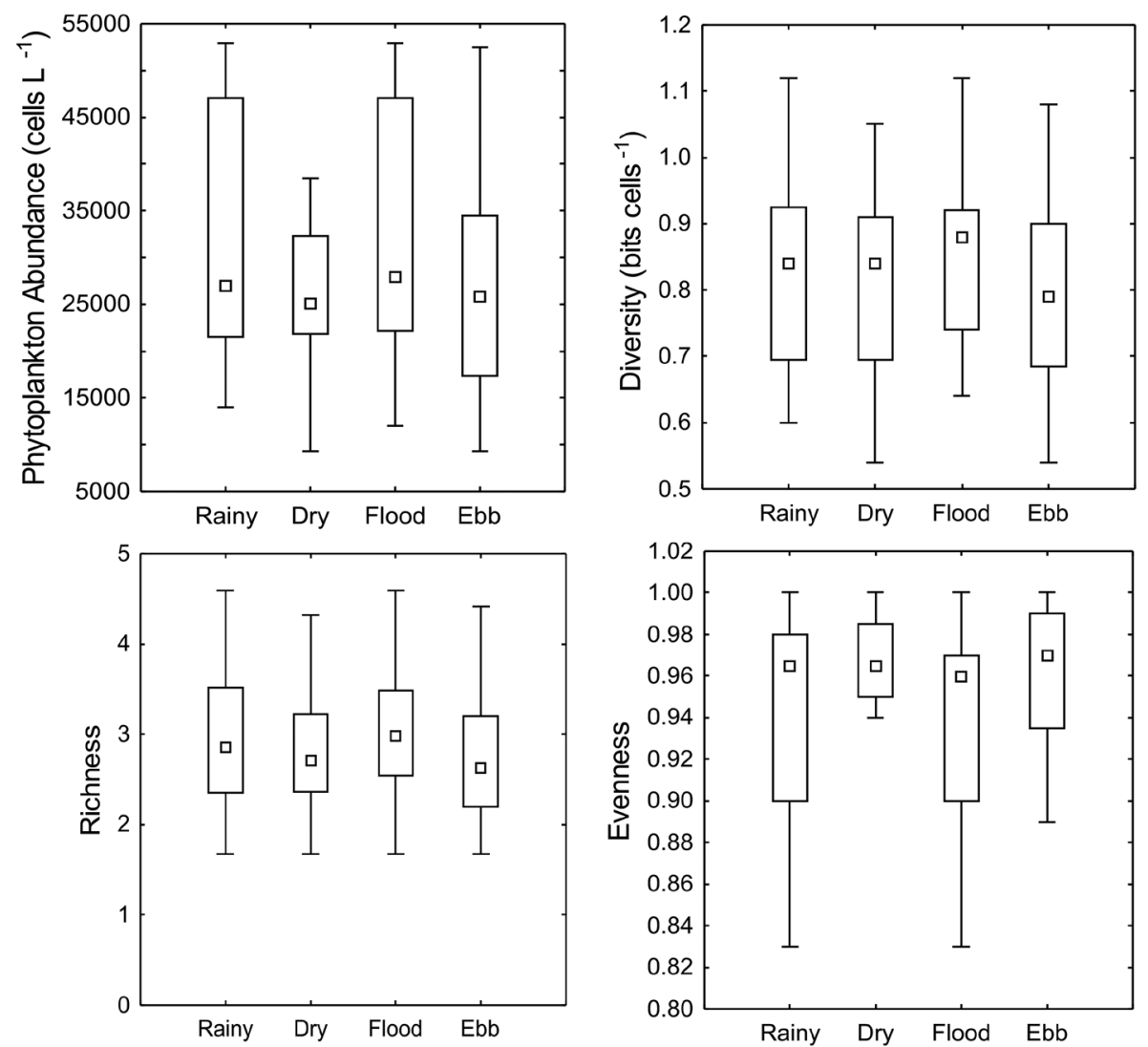

Figure 4. Boxplots of phytoplankton abundance (cells $\mathrm{L}^{-1}$ ), diversity index (bits cell ${ }^{-1}$ ), richness, and evenness in the São Marcos Bay.

Table 3. Cell abundance (cells $\mathrm{L}^{-1}$ ) and dominance index $(Y)$ of the most representative species of total phytoplankton in the São Marcos Bay. Note: F=Flood; E=Ebb.

\begin{tabular}{|c|c|c|c|c|c|c|c|c|c|c|c|c|c|}
\hline \multirow{3}{*}{ Species } & \multicolumn{6}{|c|}{ Rainy } & \multirow[b]{3}{*}{$\mathbf{Y}$} & \multicolumn{6}{|c|}{ Dry } \\
\hline & \multicolumn{2}{|c|}{ apr./10 } & \multicolumn{2}{|c|}{ jan./11 } & \multicolumn{2}{|c|}{ mar./11 } & & \multicolumn{2}{|c|}{ aug./10 } & \multicolumn{2}{|c|}{ nov./10 } & \multirow[b]{2}{*}{$\mathbf{Y}$} & \multirow[b]{2}{*}{$\Sigma$} \\
\hline & $\mathbf{F}$ & $\mathbf{E}$ & $\mathbf{F}$ & $\mathbf{E}$ & $\mathbf{F}$ & $\mathbf{E}$ & & F & $\mathbf{E}$ & $\mathbf{F}$ & $\mathbf{E}$ & & \\
\hline Nitzschia sp. & 99530 & 17525 & 13289 & 60390 & 46312 & 4039 & 0.162 & 27297 & 15853 & 16902 & 16591 & 0.064 & $\begin{array}{c}3.2 \times \\
10^{5}\end{array}$ \\
\hline Synedra sp. & 32144 & 5708 & 9874 & 18512 & 58561 & 6381 & 0.068 & 11780 & 4039 & 4258 & 15791 & 0.022 & $\begin{array}{c}1.6 x \\
10^{5}\end{array}$ \\
\hline $\begin{array}{l}\text { Diploneis } \\
\text { weissflogii }\end{array}$ & 4376 & 4011 & 3725 & 20167 & 31379 & 7742 & 0.044 & 34736 & 22753 & 13046 & 14768 & 0.062 & $\begin{array}{c}1.6 \mathrm{x} \\
10^{5}\end{array}$ \\
\hline Thalassiosira sp. & 4376 & 6017 & 5862 & 16661 & 15180 & 7405 & 0.030 & 2188 & 2188 & 5890 & 0 & 0.003 & $\begin{array}{c}6.6 \times \\
10^{4}\end{array}$ \\
\hline $\begin{array}{l}\text { Cyclotella } \\
\text { stylorum }\end{array}$ & 14002 & 3702 & 4011 & 5862 & 4012 & 6045 & 0.020 & 2188 & 0 & 4193 & 2188 & 0.002 & $\begin{array}{c}4.6 x \\
10^{4}\end{array}$ \\
\hline $\begin{array}{l}\text { Thalassiosira } \\
\text { leptopus }\end{array}$ & 0 & 0 & 10028 & 10757 & 4011 & 4039 & 0.014 & 4813 & 6564 & 4376 & 0 & 0.004 & $\begin{array}{c}4.5 \mathrm{x} \\
10^{4}\end{array}$ \\
\hline Coscinodiscus sp. & 9626 & 18543 & 4011 & 18822 & 9626 & 6381 & 0.014 & 4376 & 6227 & 0 & 2188 & 0.004 & $\begin{array}{c}7.9 x \\
10^{4}\end{array}$ \\
\hline $\begin{array}{l}\text { Eupodiscus } \\
\text { radiatus }\end{array}$ & 0 & 2006 & 0 & 2006 & 19253 & 1851 & 0.003 & 12218 & 4376 & 0 & 8751 & 0.008 & $\begin{array}{c}5.0 \times \\
10^{4}\end{array}$ \\
\hline
\end{tabular}



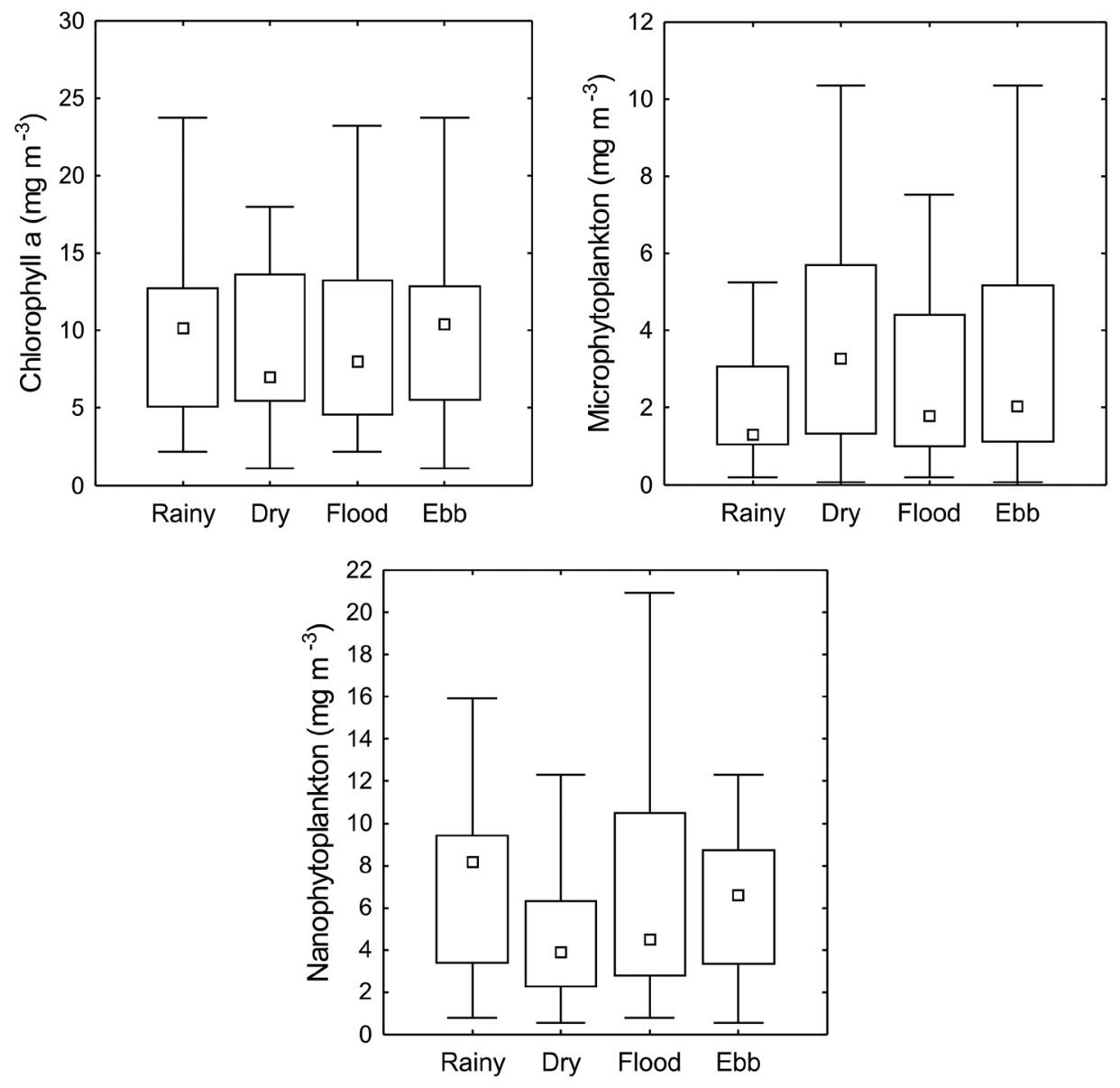

Figure 5. Seasonal and tidal variation of chlorophyll $a\left(\mathrm{mg} \mathrm{m}^{-3}\right)$, microphytoplankton $\left(\mathrm{mg} \mathrm{m}^{-3}\right)$ and nanophytoplankton $\left(\mathrm{mg} \mathrm{m}^{-3}\right)$ in the São Marcos Bay.

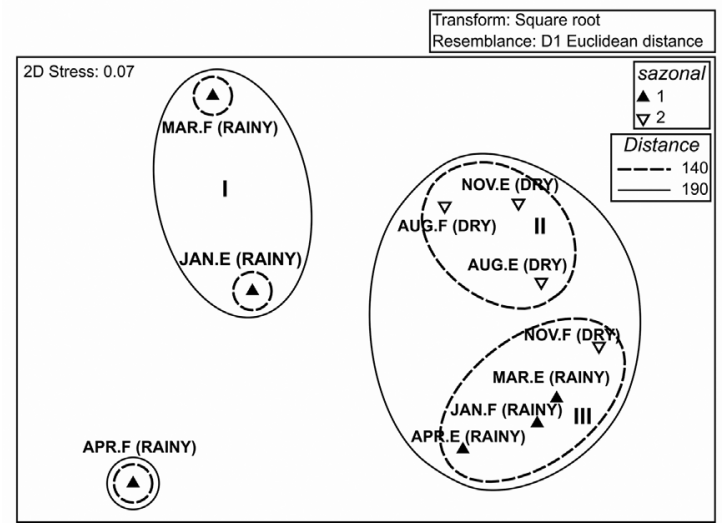

Figure 6. Representation of seasonal periods and sampling months by nMDS ordination based on phytoplankton abundance, precipitation, and salinity showing the arrangement of sampling months in three major groups (I, II, and III). Note: 1=rainy season, 2=dry season, JAN=January, APR=April, MAR=March, AUG=August, $\mathrm{NOV}=$ November and $\mathrm{F}=$ flood tide and $\mathrm{E}=\mathrm{ebb}$ tide. the variation in precipitation and salinity the data was represented as bubbles in the nMDS graph (Figure 7). The analysis showed a clear seasonal pattern with salinity values varying on account of the of precipitation regime. In response to the environmental fluctuations, the abundance of Nitzschia sp., Synedra sp., and Coscinodiscus sp. were higher in the sampling months of higher precipitation and lower salinity, while Diploneis weissflogii had higher abundance in the period of higher salinity (dry season).

The SIMPER analysis showed an overall average dissimilarity of $48.62 \%$ in relation to the discriminative species that differentiates their distribution between the rainy season and dry season. Among the discriminative species, Nitzschia sp. revealed the predominant diatom in this study with $29.12 \%$ of contribution and higher mean abundance in the rainy season $\left(4.02 \times 10^{4}\right.$ cells $\mathrm{L}^{-1}$ ) followed by Diploneis weissflogii, Synedra sp., 

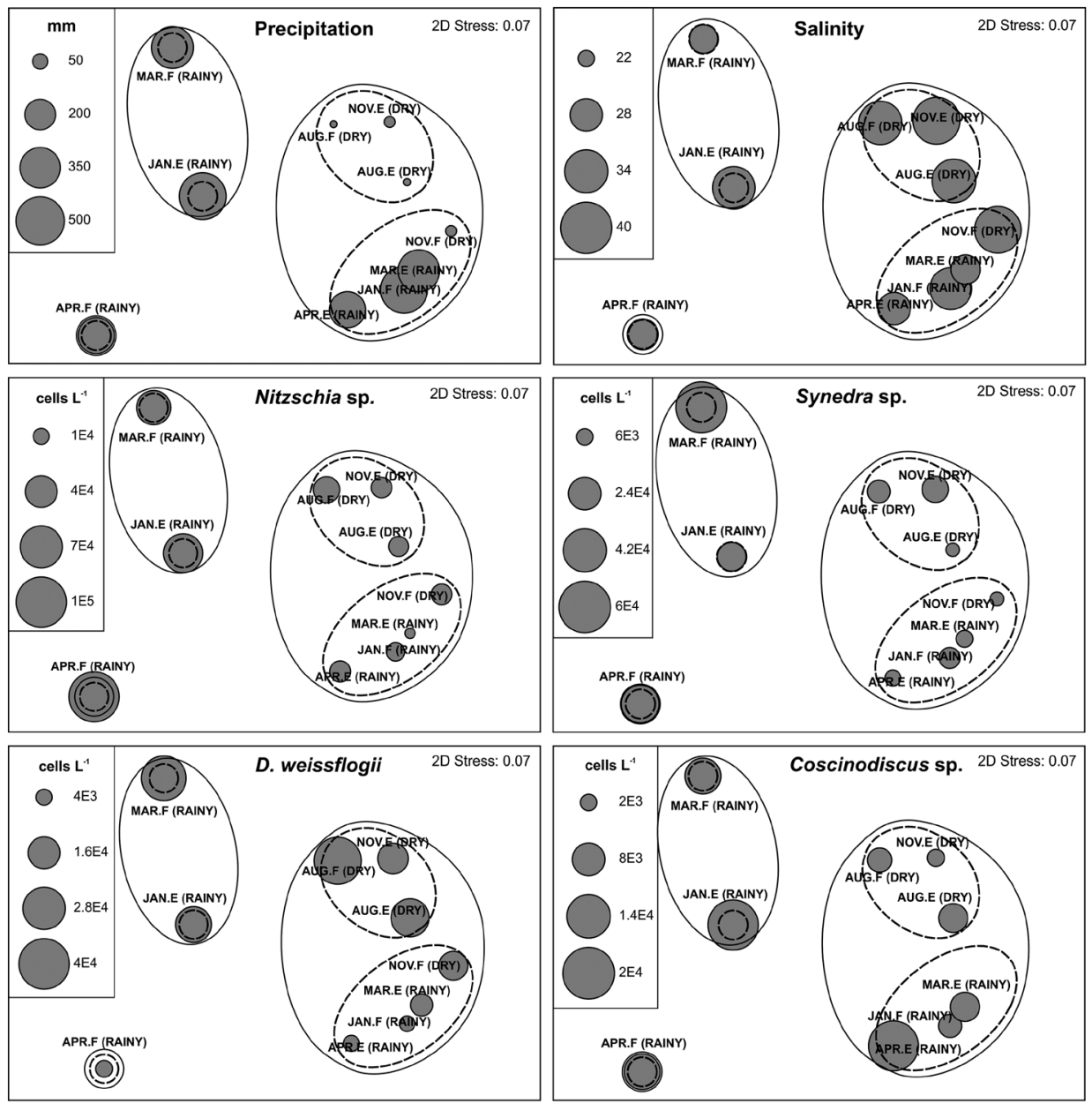

Figure 7. Bubble plot of precipitation, salinity and cell abundance (cells $\mathrm{L}^{-1}$ ) of dominant species pointed out by Dominance Index (Y) in the São Marcos Bay superimposed in the nMDS ordination.

Coscinodiscus sp. and Eupodiscus radiates, which had elevated cell abundance in the rainy season, exceptionally D. weissflogii (Table 4).

The PCA explained $51.88 \%$ of the total variance (Factor 1=3.44; Factor 2=2.28; Factor 3=1.53) showing the relation between the precipitation regime and principal variables in the São Marcos Bay (Figure 8). Factor 1 explained $24.57 \%$ of the variance, where precipitation $(0.93)$ was negatively correlated with wind speed $(-0.93)$, dissolved oxygen (-0.89), salinity (-0.71). Chlorophyll $a$ concentration (0.36) was directly correlated with turbidity $(0.80)$ and inversely correlated with Secchi depth (-0.74) and $\mathrm{pH}(-0.68)$ in the factor $2(16.34 \%)$. Phytoplankton abundance was directly correlated with $\mathrm{PO}_{4}^{-3}(0.59)$ and inversely correlated with $\mathrm{NH}_{4}^{+}(-0.65)$ in factor 3 (10.97\%).

Regarding the linear correlation, the phytoplankton abundance correlated positively with precipitation $(0.53)$ which was negatively correlated with wind speed (-0.95) and dissolved oxygen $(-0.81)$. Salinity was directly correlated with dissolved oxygen (0.62) and inversely with temperature (-0.84). The abundance of Nitzschia sp. was correlated with higher values of turbidity $(0.41)$ and lower nitrate concentrations (-0.39). Diploneis weissflogii showed relationship with dissolved oxygen (0.43). In terms of chlorophyll $a$ concentration, nanophytoplankton group showed higher correlation $(0.84)$ (Table 5).

\section{DISCUSSION}

The dynamics of phytoplankton community in tropical estuarine complex, dominated by macrotides, are influenced mainly by the seasonal conditions of the winds, tides, precipitation patterns, the discharge of rivers and the availability of nutrients; or by the combination of these factors together (Eskinazi-Leça et al., 2004; Sousa et al., 
Table 4. Principal taxa contributing to the mean dissimilarities between rainy (1) and dry (2) seasons, as determined by the SIMPER analysis.

\begin{tabular}{lccccc}
\hline Taxa & Av. dissim & Contrib. \% & Cumulative \% & Mean abund. 1 & Mean abund. 2 \\
\hline Nitszchia sp. & 14.16 & 29.12 & 29.12 & $4.02 \mathrm{E}+04$ & $1.92 \mathrm{E}+04$ \\
Diploneis weissflogii & 8.981 & 18.47 & 47.59 & $1.19 \mathrm{E}+04$ & $2.13 \mathrm{E}+04$ \\
Synedra sp. & 7.764 & 15.97 & 63.56 & $2.19 \mathrm{E}+04$ & $8.97 \mathrm{E}+03$ \\
Coscinodiscus sp. & 5.053 & 10 & 73.95 & $1.12 \mathrm{E}+04$ & $3.20 \mathrm{E}+03$ \\
Eupodiscus radiatus & 3.946 & 8.117 & 82.07 & $4.19 \mathrm{E}+03$ & $6.34 \mathrm{E}+03$ \\
Thalassiosira sp. & 3.707 & 7.625 & 89.69 & $9.25 \mathrm{E}+03$ & $2.57 \mathrm{E}+03$ \\
Thalassiosira leptopus & 2.644 & 5.437 & 95.13 & $4.81 \mathrm{E}+03$ & $3.94 \mathrm{E}+03$ \\
Cyclotella stylorum & 2.368 & 5 & 100 & $6.27 \mathrm{E}+03$ & $2.14 \mathrm{E}+03$ \\
\hline
\end{tabular}

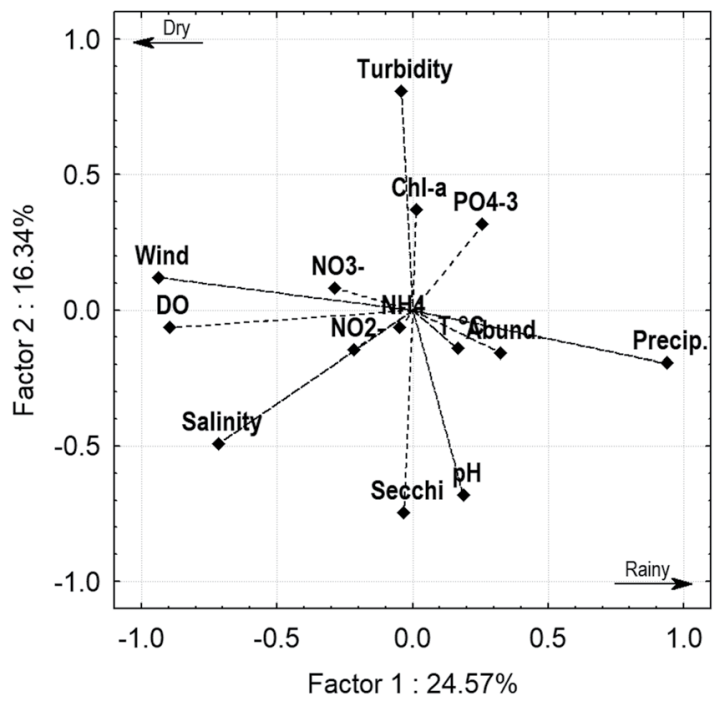

Figure 8. Principal components analysis performed on major variables in the São Marcos Bay. Note: Precip.=Precipitation; Wind=Wind speed; $\mathrm{T}{ }^{\circ} \mathrm{C}=$ Water temperature; Sal. $=$ Salinity; $\mathrm{pH}$; Secchi $=$ Secchi depth; Turb.=Turbidity; $\mathrm{DO}=$ Dissolved oxygen; $\mathrm{NH}_{4}^{+}=$ammonium concentrations; $\mathrm{NO}_{2}^{-}=$Nitrite concentrations; $\mathrm{PO}_{4}^{-3}=$ Orthophosphate concentrations; $\mathrm{NO}_{3}^{-}=$Nitrate concentrations; $\mathrm{Chl}-\mathrm{a}=$ Chlorophyll $a$ and Abund. $=$ Phytoplankton abundance.

2009; Chowdhury et al., 2017). In the São Marcos Bay, the well-defined precipitation regime is an important regulator of phytoplankton structure and chlorophyll $a$ as well as some hydrological variables, for example, associated with the river discharge increasing may contribute mainly to salinity, dissolved oxygen and nutrients lowering.

The precipitation regime in the state of Maranhão is controlled by the Intertropical Convergence Zone (ITCZ), which produces the highest rainfall in northeastern Brazil between the months of January and May. The ITCZ, during the following months of the year, is much less intense and results in a dry season (Dias et al., 2016; Funceme, 2016). This seasonal pattern was confirmed along the study period and it was in accordance with previous studies for the northeastern region (Azevedo et al., 2008; Rodrigues and Cutrim, 2010; Duarte-dos-Santos et al., 2017).

In the São Marcos Bay, the salinity showed a clear seasonal variation with values lower in the period of higher precipitation and high phytoplankton abundance. In tropical coastal systems, salinity acts as an ecological barrier and regulator for aquatic life distribution, since temperature and sunlight have slight variation through the year (Santos-Fernandes et al., 1998; Attrill and Rundle, 2002). In addition, freshwater influx, seawater incursion and industrial as well as domestic effluent discharges may result in well-marked longitudinal gradients of salinity and nutrients that led to significant spatio-temporal changes in the structure of the phytoplankton community (Chowdhury et al., 2017).

The spread TS diagram analysis for the sampling period reveals an increase in salinity observed during the dry season. It can be explained by the fluvial flux decreasing (Mearim and Pindaré Rivers) and marine waters influence on the São Marcos Bay, which is probably due to the decrease in the aspect ratio determined by free surface variation and surface gravity wave propagation (Dias et al., 2013).

The variation of dissolved oxygen obtained in the bay are common to marine systems of Maranhense coast in natural conditions (Azevedo et al., 2008; Rodrigues and Cutrim, 2010). It may be explained by the tidal regime (macrotides) and consequently high local hydrodynamic that combined can accentuate the ocean-atmosphere interface and the oxygenation of the water (Monteiro et al., 2009; Matos et al., 2016). Furthermore, precipitation influences the fluctuations in the oxygen dissolved, because can lead to the increase of dead organic matter decreasing its concentration during the oxidation process (Kress et al., 2002). The undersaturation found in the bay did not indicate evidence of eutrophication events and polluted 


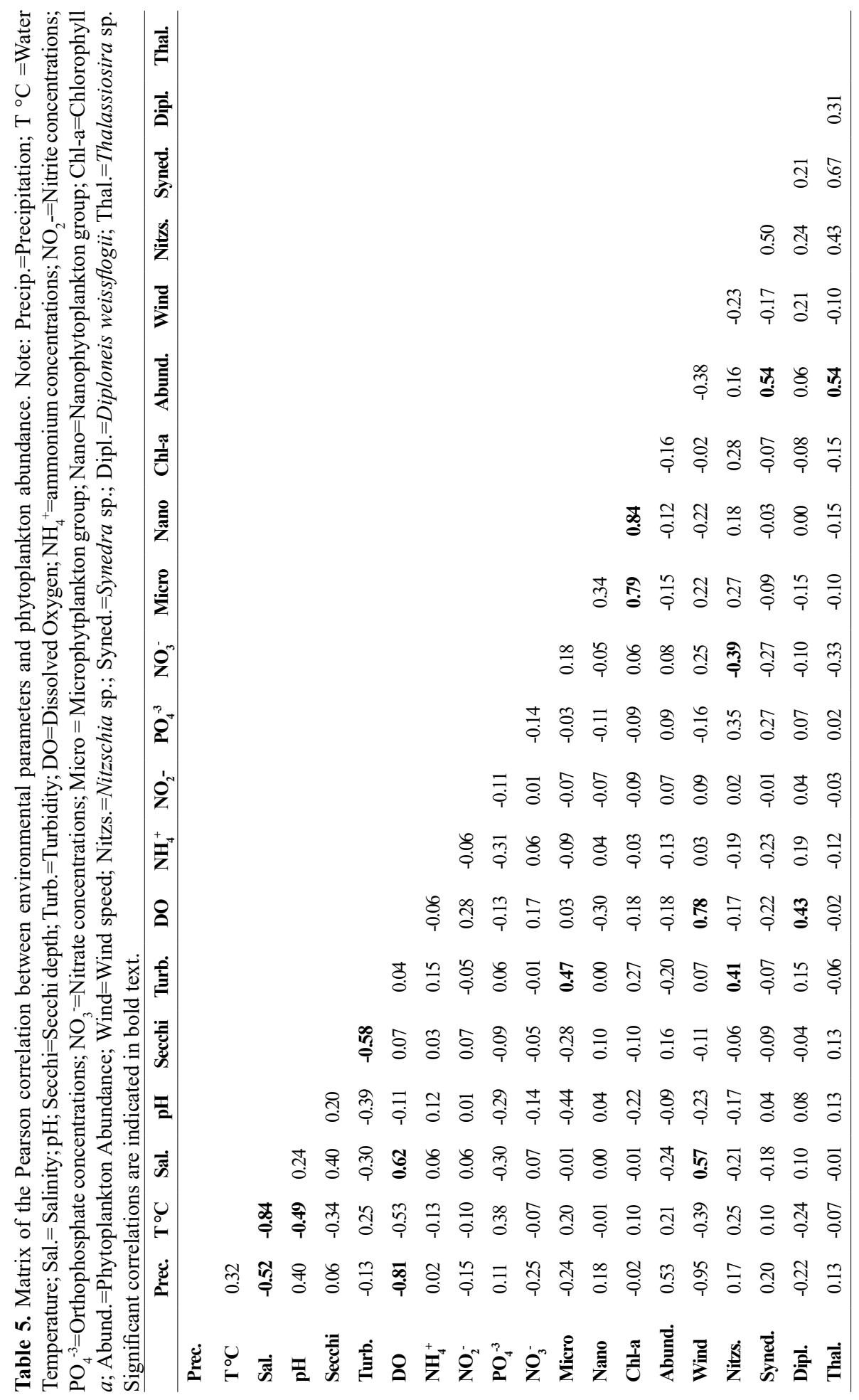


zones which is expected in urbanized areas (Macêdo and Costa, 1978). Seasonal variation of dissolved oxygen was also observed in other tropical estuaries (Borges et al., 2012; Otsuka et al., 2016).

In estuarine systems, light availability and water turbidity can limit phytoplankton growth (Demers et al., 1986; Domingues et al., 2011). In the São Marcos Bay, high turbidity results in a narrow euphotic zone due to sunlight attenuation with photosynthetic process (chlorophyll $a$ ) restricted to surface layers (Teixeira et al., 1988; Azevedo et al., 2008). Turbidity of water is usually caused by the presence of suspended matter (organic and inorganic matter) in water column that may decrease the light intensity and affect the phytoplankton development. Nevertheless, the elevated turbidity associated with high nutrient loading may enhance the growth of phytoplankton in high hydrodynamic systems (Rai and Rajashekhar, 2014).

In this study, turbidity showed a positive relationship with chlorophyll $a$, wind and tidal currents (macrotides). This relationship indicates an intense estuarine mixing, which promotes high resuspension of sediments and ticopelagic species such as Nitzschia sp. with high abundance along the present study. In terms of contribution of phytoplankton biomass (chlorophyll $a$ ), the autotrophic plankton was mainly composed by nanopicoplankton. This fact was also reported by Guenther et al. (2012), Kocum and Sutcu (2014), and Sathicq et al. (2017). Guenther and Bozelli (2004) state that as small cells show high surface/volume ratio, they are capable of remaining in suspension longer and the lateral transport did not become size-selective.

In general, riverine nutrients are essential sources of production of phytoplankton in coastal areas (Sin et al., 2015). Variation in nitrate and its reduced inorganic compounds are predominantly the results of biologically activated reactions (Kathiravan et al., 2013). Usually, high levels of nitrate are recorded in the rainy period (Symader and Bierl, 1998). It is due to the runoff and enrichment of terrogenous deposit with a lot of nutrients, together with high temperature and water mixing might favor the nitrate replenishment mechanism (Kalaiarasi et al., 2012). The efficient assimilation by phytoplankton and enhancement by surface runoff results in large scale spatio-temporal variation of nitrate in the coastal areas (Noriega et al., 2005).
The characterization of phytoplankton communities has become an important tool to understand the functionality of ecosystems (Soria-Píriz et al., 2017; Santana et al., 2018). The phytoplankton community composition in the São Marcos Bay revealed diatoms as the most successful phytoplankton group (e.g., growth and abundance). The wild occurrence of diatoms in coastal ecosystems is based on their euryhaline nature and affinity for favorable high nutrient environments (Paiva et al., 2006; Rodrigues and Cutrim, 2010; Zhou et al., 2016).

Depending upon the adaptability to salinity gradients, euryhaline species, which occur throughout estuarine systems in varying density, and stenohaline species can show elevate abundance when the environmental conditions are favorable (Chowdhury et al., 2017). The presence of some riverine phytoplankton in the rainy season shows the succession through the seasons (Honorato da Silva et al., 2009; Santiago et al., 2010) and its importance to the bulk biomass, due to the increase of river discharge. However, the dominance of diatoms Nitzschia sp., Diploneis weissflogii and Synedra sp. confirms their capacity to be successful in dynamic environments.

\section{CONCLUSIONS}

The high hydrodynamics found in the São Marcos Bay, governed by macrotidal regimes, may be considered as the main driving force that leads to an ecological stability scenario for the phytoplankton community. The precipitation regime was also an important factor capable of driving favorable hydrological conditions to phytoplankton development, promoting seasonal variations in terms of salinity, dissolved oxygen, turbidity, and nutrient concentrations. Hence, the composition of phytoplankton community dominated by diatoms showed Nitzschia sp., Diploneis weissflogii and Synedra sp. as the most representative species and confirmed their capacity to be successful in dynamic environments such as macrotidal estuaries. In addition, the phytoplankton biomass characterized the bay as quite productive with the nanophytoplankton as the dominant fraction in both seasons and tides. Thus, these results are supported by high hydrodynamics associated to precipitation regime of the São Marcos Bay and consequently, does not allow the rapid phytoplankton growth that could contribute to the eutrophication processes and poor water quality. 


\section{ACKNOWLEDGEMENTS}

We thank the grant given by PRÓ-VIDA Brazil Company that financed this present study. The authors would like to thank the FAPEMA Foundation (Fundação de Amparo à Pesquisa e ao Desenvolvimento Científico e Tecnológico do Maranhão), UEMA (Universidade Estadual do Maranhão) and UFMA (Universidade Federal do Maranhão) for the support and also express special gratitude to Anna O'Reilly, Patrick O'Reilly and the professor Rose Puskar for the careful English correction.

\section{REFERENCES}

AMARAL, R. F. \& ALFREDINI, P. 2010. Modelação hidrossedimentológica no canal de acesso do complexo portuário do Maranhão. Revista Brasileira de Recursos Hídricos, 15, 5-14. DOI: $10.21168 /$ rbrh.v15n2

APHA (American Public Health Association). 2012. Standard methods for the examination of water and wastewater. $22^{\text {nd }}$ ed. Washington: American Public Health Association, American Water Works Association, Water Environment Federation.

ATTRILL, M. J. \& RUNDLE, S. D. 2002. Ecotone or ecocline: ecological boundaries in estuaries. Estuarine, Coastal and Shelf Science, 55, 929-936. DOI: https://doi.org/10.1006/ ecss.2002.1036

AZEVEDO, A. C. G., FEITOSA, F. A. N. \& KOENING, M. L. 2008. Distribuição espacial e temporal da biomassa fitoplanctônica e variáveis ambientais no Golfão Maranhense, Brasil. Acta Botanica Brasilica, 22, 870-877. DOI: http:// dx.doi.org/10.1590/S0102-33062008000300022

AZHIKODAN, G. \& YOKOYAMA, K. 2016. Spatio-temporal variability of phytoplankton (Chlorophyll- $a$ ) in relation to salinity, suspended sediment concentration, and light intensity in a macrotidal estuary. Continental Shelf Research, 126, 1526. DOI: https://doi.org/10.1016/j.csr.2016.07.006

BAZIN, P., JOUENNE, F., DETON-CABANILLAS, A. F., PÉREZ-RUZAFA, Á. \& VÉRON, B. 2014. Complex patterns in phytoplankton and microeukaryote diversity along the estuarine continuum. Hydrobiologia, 726, 155-178. DOI: $10.1007 / \mathrm{s} 10750-013-1761-9$

BORGES, G. C. P., SILVA-CUNHA, M. G. G., SANTIAGO, M. F. \& LIMA, J. C. 2012. Comunidade fitoplanctônica e variáveis ambientais em área portuária, nordeste do Brasil. Tropical Oceanography, 40, 309-318. DOI: 10.5914/tropocean. v40i2.5408

BURFORD, M. A., ALONGI, V., MCKINNON, A. D. \& TROTT, L. A. 2008. Primary production and nutrients in a tropical macrotidal estuary, Darwin Harbour, Australia. Estuarine, Coastal and Shelf Science, 79, 440-448. DOI: https://doi. org/10.1016/j.ecss.2008.04.018

CARSTENSEN, J., SÁNCHEZ-CAMACHO, M., DUARTE, C. M., KRAUSE-JENSEN, D. \& MARBÀ, N. 2011. Connecting the Dots: Responses of Coastal Ecosystems to Changing Nutrient Concentrations. Environmental Science \& Technology, 45, 9122-9132. DOI: 10.1021/es202351y
CHAI, C., JIANG, T., CEN, J., GE, W. \& LU, S. 2016. Phytoplankton pigments and functional community structure in relation to environmental factors in the Pearl River Estuary. Oceanologia, 58, 201-211. DOI: https://doi.org/10.1016/j. oceano.2016.03.001

CHOWDHURY, M., HARDIKAR, R., KESAVAN, H. C., THOMAS, J., MITRA, A., ROKADE, M. A., NAIDU, V. S. \& SUKUMARAN, S. 2017. Nutrient stoichiometry and freshwater flow in shaping of phytoplankton population in a tropical monsoonal estuary (Kundalika Estuary). Estuarine, Coastal and Shelf Science, 198, 73-91. DOI: http://dx.doi. org/10.1016/j.ecss.2017.08.019

CLOERN, J. E. 2001. Our evolving conceptual model of the coastal eutrophication problem. Marine Ecology Progress Series, 210, 223-253. DOI: https://doi.org/10.3354/meps210223

CLOERN, J. E., FOSTER, S. Q. \& KLECKNER, A. E. 2014. Phytoplankton primary production in the world's estuarine-coastal ecosystems. Biogeosciences, 11, 2477-2501. DOI:10.5194/bg-11-2477-2014

COSTANZA, R., D’ARGE, R., DE GROOT, R., FARBER, S., GRASSO, M., HANNON, B., LIMBURG, K., NAEEM, S., O'NEILL, R. V., PARUELO, J., RASKIN, R. G., SUTTON, P. \& VAN DEN BELT, M. 1997. The value of the world's ecosystem services and natural capital. Nature, 387, 253-260.

DAJOZ, R. 1983. Ecologia geral, Petrópolis, Vozes.

DEMERS, S., LEGENDRE, L. \& THERRIAULT, J. C. 1986. Phytoplankton responses to vertical tidal mixing. In: BOWMAN, M. J., YENTSCH, C. M. \& PETERSON, W. T (eds.) Tidal Mixing and Plankton Dynamics. New York: Springer. $1-40$.

DIAS, F. J. S., CASTRO, B. M. \& LACERDA, L. D. 2013. Continental shelf water masses off the Jaguaribe River (4S), northeastern Brazil. Continental Shelf Research, 66, 123-135. DOI: https://doi.org/10.1016/j.csr.2013.06.005

DIAS, F. J. S., CASTRO, B. M., LACERDA, L. D., MIRANDA, L. B. \& MARINS, R. V. 2016. Physical characteristics and discharges of suspended particulate matter at the continent-ocean interface in an estuary located in a semiarid region in northeastern Brazil. Estuarine, Coastal and Shelf Science, 180, 258-274. DOI: https://doi.org/10.1016/j. ecss.2016.08.006

DOMINGUES, R. B., ANSELMO, T. P, BARBOSA, A. B., SOMMER, U. \& GALVÃO, H. M. 2011. Light as a driver of phytoplankton growth and production in the freshwater tidal zone of a turbid estuary. Estuarine, Coastal and Shelf Science, 91, 526-535. DOI: https://doi.org/10.1016/j.ecss.2010.12.008

DUARTE DOS SANTOS, A. K., OLIVEIRA, A. L. L., FURTADO, J. A., FERREIRA, F. S., ARAÚJO, B. O., CORRÊA, J. J. M., CAVALCANTI, L. F., AZEVEDO CUTRIM, A. C. G. \& CUTRIM, M. V. J. 2017. Spatial and seasonal variation of microphytoplankton community and the correlation with environmental parameters in a hypereutrophic tropical estuary - Maranhão - Brazil. Brazilian Journal of Oceanography, 65, 356-372. DOI: 10.1590/S1679-87592017134406503

EL-ROBRINI, M., MARQUES, V. J., SILVA, M. A., EL-ROBRINI, M. H. S., FEITOSA A., TAROUCO, J. E. F., SANTOS, J. H. S. \& VIANA, J. R. 2006. Atlas de erosão e progradação da zona costeira do estado do maranhão - região amazônica: área oceânica e estuarina. In: MUEHE, D. (org.) Atlas de erosão e progradação da zona costeira brasileira. São Paulo. 
ESKINAZI-LEÇA, E., KOENING, M. L. \& SILVA-CUNHA, M. G. G. 2004. Estrutura e dinâmica da comunidade fitoplanctônica. In: ESKINAZI-LEÇA, E., NEUMANN-LEITÃO, S. \& COSTA, M. F. (eds.) Oceanografia: um cenário tropical. Recife: Bagaço.

FERRARIO, M., SAR, E. \& SALA, S. 1995. Metodologia básica para el estudio de fitoplancton com especial referencia a las diatomeas. In: ALVEAL, K., FERRARIO, M. E., OLIVEIRA, E. C. \& SAR, E. (eds.) Manual de métodos ficológicos. Concepción: Universidad de Concepción.

FUNCEME, 2016. Sistemas meteorológicos causadores de chuva na região nordeste do Brasil [cited 2017 Dec 5]. Available from: http://www.funceme.br/produtos/script/chuvas/Grafico_chuvas_postos_pluviometricos/entender/entender2.htm

GONZÁLEZ-GORBEÑA, E., ROSMAN, P. C. C. \& QASSIM, R. Y. 2015. Assessment of the tidal current energy resource in São Marcos Bay, Brazil. Journal of Ocean Engineering and Marine Energy, 1, 421-433. DOI: 10.1007/s40722-015-0031-5

GUENTHER, M., LIMA, I., MUGRABE, G., TENENBAUM, D. R., GONZALEZ-RODRIGUEZ, E. \& VALENTIN, J. L. 2012. Small time scale plankton structure variations at the entrance of a tropical eutrophic bay (Guanabara Bay, Brazil). Brazilian Journal of Oceanography, 60, 405-414. DOI: http:// dx.doi.org/10.1590/S1679-87592012000400001

GUENTHER, M. \& BOZELLI, R. 2004. Factors influencing algae-clay aggregation. Hydrobiologia, 523, 217-223.

GUIRY, M. D. \& GUIRY, G. M. 2017. Algae Base. World-wide electronic publication, National University of Ireland, Galway [cited 2017 Dec 5]. Available from: http://www.algaebase.org

HONORATO DA SILVA, M., SILVA-CUNHA, M. G. G., PASSAVANTE, J. Z. O., GREGO, C. K. S. \& MUNIZ, K. 2009. Estrutura sazonal e espacial do microfitoplâncton no estuário tropical do rio Formoso, PE, Brasil. Acta Botânica Brasílica, 23, 335-368.

KALAIARASI, M., PAUL, P., LATHASUMATHI, C. \& STELLA, C. 2012. Seasonal variations in the physico-chemical characteristics of the two coastal waters of Palk-Strait in Tamil Nadu, India. Global Journal of Environmental Research, 6, 66-74.

KATHIRAVAN, T., SUNDARAMANICKAM, A., VARADHARAJAN, D. \& BALASUBRAMANIAN, T. 2013. Seasonal variations in physico-chemical parameters of Parangipettai coast, South east coast of India. International Journal of Pharmaceutical and Biological Archive, 4, 240-249.

KATSIAPI, M., MOUSTAKA-GOUNI, M. \& SOMMER, U. 2016. Assessing ecological water quality of freshwaters: PhyCoI-a new phytoplankton community Index. Ecological Informatics, 31, 22-29. DOI: https://doi.org/10.1016/j. ecoinf.2015.11.004

KATZ, M. E., FINKEL, Z. V., GRZEBYK, D., KNOLL, A. H. \& FALKOWSKI, P. G. 2004. Evolutionary trajectories and biogeochemical impacts of marine eukaryotic phytoplankton. Annual Review Ecology Evolution Systematics, 35, 523-556.

KOCUM, E. \& SUTCU, A. 2014. Analysis of variations in phytoplankton community size-structure along a coastal trophic gradient. Journal of Coastal Research, 30, 777-784. DOI: https://doi.org/10.2112/JCOASTRES-D-12-00045.1

KRESS, N., COTO, S. L., BRENES, C. L., BRENNER, S. \& ARROYO, G. 2002. Horizontal transport and seasonal distribution of nutrients, dissolved oxygen and chlorophyll-a in the Gulf of Nicoya, Costa Rica: A tropical estuary. Continental Shelf Research, 22, 51-66.
LAVÔR-FERNANDES, G. 1988. Microfitoplâncton da Baía de São Marcos, São Luís, Maranhão, Brasil. Gayana, 45, 265 274.

MACÊDO, S. J. \& COSTA, K. M. P. 1978. Estudos ecológicos da região de Itamaracá. Pernambuco - Brasil. Condições Hidrológicas do Estuário do Rio Botafogo. Ciência e Cultura, 30, 346-368.

MAMAYEV, O. I. 1975. Temperature-Salinity Analysis of World Ocean Waters, Amsterdam, Elsevier Scientific.

MARGALEF, R. 1958. Temporal succession and spatial heterogeneity in phytoplankton. In: BUZZATI-TRAVERSO, A. A. (ed.) Perspectives in Marine Biology. Berkeley: California University Press.

MATOS, J. B., OLIVEIRA, S. M. O., PEREIRA, L. C. C., \& COSTA, R. M. 2016. Structure and temporal variation of the phytoplankton of a macrotidal beach from the Amazon coastal zone. Anais da Academia Brasileira de Ciências, 88, 1325-1339. DOI: http://dx.doi.org/10.1590/00013765201620150688

MIRANDA, L. B. 1985. Forma da correlação T-S de massas de água das regiões costeira e oceânica entre cabo de São Tome (RJ) e a Ilha de São Sebastião (SP). Boletim do Instituto Oceanográfico, 33, 105-119. DOI: http://dx.doi.org/10.1590/ S0373-55241985000200002

MONTEIRO, M. C., PERREIRA, C. C. L. \& OLIVEIRA, S. M. O. 2009. Morphodynamic changes of a macrodital sand beach in the Brazilian Amazon coast (Ajuruteua-Pará). Journal of Coastal Research, SI 56 (Proceedings of the $10^{\text {th }}$ International Coastal Symposium), 103-107.

MORAIS, J. O. 1977. Processos de sedimentação na baía de São Marcos, estado do Maranhão, Brasil. Arquivos de Ciências do Mar, 17, 153-164.

NORIEGA, C. D., COSTA, K. M. P., FEITOSA, F. A. N., FLORES-MONTES, M. J., GREGO, C. K. S., SOARES, G. S. S. \& SILVA, H. P. 2005. Distribuição espacial da biomassa fitoplanctônica e sua relação com os sais nutrientes, no sistema estuarino de Barra das Jangadas (Pernambuco - Brasil). Arquivos de Ciências do Mar, 38, 5-18.

OTSUKA, A. Y., FEITOSA, F. A. N., FLORES-MONTES, M. J. \& SILVA, A. 2016. Dynamics of Chlorophyll a and Oceanographic Parameters in the Coastal Zone: Barra das Jangadas-Pernambuco, Brazil. Journal of Coastal Research, 32, 490-499. DOI: https://doi.org/10.2112/JCOASTRESD-14-00135.1

PAERL, H. W., VALDES, L. M., PINCKNEY, J. L., PIEHLER, M. F., DYBLE, J. \& MOISANDER, P. H. 2003. Phytoplankton Photopigments as Indicators of Estuarine and Coastal Eutrophication. BioScience, 53, 953-964. DOI: /10.1641/0006-3568(2003)053[0953:PPAIOE]2.0.CO;2

PAERL, H. W., ROSSIGNOL, K. L., HALL, S. N., PEIERLS, B. L. \& WETZ, M. S. 2010. Phytoplankton community indicators of short and long-term ecological change in the anthropogenically and climatically impacted Neuse River estuary, North Carolina, USA. Estuaries and Coasts, 33, 485-497. DOI: $10.1007 / \mathrm{s} 12237-009-9137-0$

PAIVA, R. S., ESKINAZI-LEÇA, E., PASSAVANTE, J. Z. O., SILVA-CUNHA, M. G. G. \& MELO, N. F. A. C. 2006. Considerações ecológicas sobre o fitoplâncton da baía do Guajará e foz do rio Guamá, Pará, Brasil. Boletim do Museu Paraense Emílio Goeldi. Ciências Naturais, 1, 133-146. 
PARSONS, T. R. \& STRICKLAND, J. D. H. 1963. Discussion of Spectrophotometric Determination of Marine-Plant Pigments, with Revised Equations for Ascertaining Chlorophylls and Carotenoids. Journal of Marine Research, 21, 155-163.

PIELOU, E. C. 1966. Species-diversity and pattern-diversity in the study of ecological succession. Journal of Theoretical Biology, 10, 370-383. DOI: https://doi.org/10.1016/00225193(66)90133-0

RAI, S. V. \& RAJASHEKHAR, M. 2014. Seasonal assessment of hydrographic variables and phytoplankton community in the Arabian Sea Waters of Kerala, Southwest Coast of India. Brazilian Journal of Oceanography, 62, 279-289. DOI: 10.1590/ s1679-87592014069906204

RIOS, L. 2001. Estudos de geografia do Maranhão. $3^{\mathrm{a}}$ ed. São Luís: Graphis.

RODRIGUES, E. I. \& CUTRIM, M. V. J. 2010. Relações entre as variáveis físicas, químicas e fitoplanctônicas de três áreas estuarinas da costa norte do Brasil - São José de Ribamar, Cedral e Cajapió, MA. Arquivos Ciências do Mar, 43, 45-54.

SANTANA, R. M. C., DOLBETH, M., BARBOSA, J. E. L. \& PATRÍCIO, J. 2018. Narrowing the gap: Phytoplankton functional diversity in two disturbed tropical estuaries. Ecological Indicators, 86, 81-93.

SANTIAGO, M. F., SILVA-CUNHA, M. G. G., NEUMANN-LEITÃO, S., COSTA, K. M. P., PALMEIRA, G. C. B., PORTO NETO, F. F. \& NUNES, F. S. 2010. Phytoplankton dynamics in a highly eutrophic estuary in tropical Brazil. Brazilian Journal of Oceanography, 58, 189-205. DOI: 10.1590/ S1679-8759201000030000

SANTOS-FERNANDES, T. L., PASSAVANTE, J. Z. O, KOENING, M. L. \& MACÊDO, S. J. 1998. Fitoplâncton do estuário do rio Jaguaribe, (Itamaracá, Pernambuco, Brasil): Biomassa. Trabalhos Oceanográficos da Universidade Federal de Pernambuco (Recife), 26, 1-18.

SATHICQ, M. B., GÓMEZ, N., BAUER, D. E. \& DONADELLI, J. 2017. Use of phytoplankton assemblages to assess the quality of coastal waters of a transitional ecosystem: Río de la Plata Estuary. Continental Shelf Research, 150, 10-17. DOI: https://doi.org/10.1016/j.csr.2016.08.009.

SHANNON, C. E. 1948. A mathematical theory of communication. Bulletin of System Technology Journal, 27, 379-423.

SIN, Y., LEE, E., LEE, Y. \& SHIN, K. 2015. The river-estuarine continuum of nutrients and phytoplankton communities in an estuary physically divided by a sea dike. Estuarine, Coastal and Shelf Science, 163, 279-289. DOI: https://doi. org/10.1016/j.ecss.2014.12.028
SORIA-PÍRIZ, S. GARCÍA-ROBLEDO, E. PAPASPYROU, S. AGUILAR, V. SEGURO, I. ACUÑA, J. MORALES, Á. CORZO, A. 2017. Size fractionated phytoplankton biomass and net metabolism along a tropical estuarine gradiente. Limnology Oceanography, 62, 309-326.

SOUSA, E. B., COSTA, V. B., PEREIRA, L. C. C., \& COSTA, R. M. 2009. Variação temporal do fitoplâncton e dos parâmetros hidrológicos da zona de arrebentação da Ilha Canela (Bragança, Pará, Brasil). Acta Botanica Brasilica, 23, 1084-1095. DOI: http://dx.doi.org/10.1590/S0102-33062009000400018

SOUZA-FILHO, P. W. M., SALES, M. E. C., PROST, M. T. R. C., COSTA, F. R. \& SOUZA, L. F. M. O. 2005. Zona Costeira Amazônica: $\mathrm{O}$ cenário regional e os indicadores bibliométricos em C\&T. In: SOUZA FILHO, P. W. M., CUNHA, E. R. S. P., SALES M. E. C., SOUZA, L. F. M. O. \& COSTA, F. R. (org.) Bibliografia da Zona Costeira Amazônica. Belém: Museu Paraense Emilio Goeldi/Universidade Federal do Pará/ Petrobras.

SUN, J., LIU, D. Y., GAO, H. W. \& HAN, X. T. 2004. Phytoplankton Community of the Bohai Sea in winter 2001. Journal of Ocean University of Qingdao, 34, 413-422.

SYMADER, W. \& BIERL, R. 1998. The heterogeneity of runoff and its significance for water quality problems. Hydrological Sciences Journal, 43, 103-113.

TEIXEIRA, C., ARANHA, F. J., BARBIERI, R., \& MELO, O. T. 1988. Produção primária e clorofila a do fitoplâncton e parâmetros físicos e químicos do estreito dos Coqueiros, Maranhão, Brasil. Revista Brasileira de Biologia, 48, 29-39.

UNESCO. 1966. Determination of photosynthetic pigments in sea waters. Report of SCOR/UNESCO working group 17 with meat from 4 to 6 June 1964. (Monographys on Oceanology Methodology), Paris, UNESCO.

VILLAFAÑE, V. E. \& REID, F. M. H. 1995. Métodos de microscopia para la cuantificación del fitoplancton. In: ALVEAR, K., FERRARIO, M. E., OLIVEIRA, E. C. \& SAR, E. (eds.) Manual de métodos ficológicos. Concepción: Universidad de Concepción.

ZHOU, W., GAO, J., LIAO, J., SHI, R., LI, T., GUO, Y. \& LONG, A. 2016. Characteristics of phytoplankton biomass, primary production and community structure in the Modaomen Channel, Pearl River Estuary, with special reference to the influence of saltwater intrusion during neap and spring tides. PLoS ONE, 11, e0167630. DOI:10.1371/journal. pone.0167630 